\title{
Association schemes related to Delsarte-Goethals codes
}

\author{
Sihuang Hu • Tao Feng • Gennian Ge
}

Received: 20 December 2012 / Accepted: 7 January 2014 / Published online: 22 January 2014

(C) Springer Science+Business Media New York 2014

\begin{abstract}
In this paper, we construct an infinite series of 9-class association schemes from a refinement of the partition of Delsarte-Goethals codes by their Lee weights. The explicit expressions of the dual schemes are determined through direct manipulations of complicated exponential sums. As a byproduct, another three infinite families of association schemes are also obtained as fusion schemes and quotient schemes.
\end{abstract}

Keywords Association schemes · Gray map · Quaternary codes · Delsarte-Goethals codes

\section{Introduction}

Since the discovery of the $\mathbb{Z}_{4}$-linearity of Kerdock, Preparata, Goethals, and Delsarte-Goethals codes (see [10]), there have been several applications of $\mathbb{Z}_{4}$-linear codes in the constructions of combinatorial configurations, such as $t$-designs and association schemes. According to the paper [22] of Solé, the discovery of the $\mathbb{Z}_{4}$ linearity is motivated by a construction of association schemes due to Liebler and Mena (see [17]) using Galois rings of characteristic 4. The research on constructing $t$-designs from linear codes over $\mathbb{Z}_{4}$ is first put forward by Harada in [11] and subsequently followed by Helleseth and his collaborators in a series of papers (see [21] and the references therein).

\footnotetext{
S. Hu · T. Feng

Department of Mathematics, Zhejiang University, Hangzhou, 310027 Zhejiang, China

G. Ge (凶)

School of Mathematical Sciences, Capital Normal University, Beijing 100048, China e-mail: gnge@zju.edu.cn
}

T. Feng · G. Ge

Beijing Center for Mathematics and Information Interdisciplinary Sciences, Beijing 100048, China 
Association schemes form a central part of algebraic combinatorics and have played important roles in several branches of mathematics, such as coding theory and graph theory. Henry Cohn and his collaborators in [2] conjectured that a certain 3 -class association scheme on 64 points determines a universally optimal configuration in $\mathbb{R}^{14}$. Then Abdukhalikov, Bannai, and Suda [1] generalized this example in terms of binary and quaternary Kerdock and Preparata codes, as well as in terms of maximal set of mutually unbiased bases. To be specific, they constructed a series of 3-class association schemes using the partition of shortened Kerdock codes induced by their Lee weights, and dual schemes on the cosets of punctured Preparata codes. This motivates us to look at another important class of quaternary codes, the Delsarte-Goethals $(\mathcal{D G})$ codes.

The $\mathcal{D G}$ code has six nonzero Lee weights. It turns out that the partition of the $\mathcal{D G}$ code by its Lee weights does not give an association scheme. We should further pick out the codewords of the Kerdock code, and this eventually yields a 9-class association scheme. Using complicated exponential sums and heavy computations, we get the structure of the dual scheme and their eigenmatrices. The dual scheme cannot be obtained in an analogous way on the cosets of the Goethals code. There is a conjectured 22-class association scheme on the $\mathcal{D G}$ code by the partition using its complete weight enumerator, but our scheme is not a fusion scheme of this conjectured scheme.

When $m=3$, the image of the $\mathcal{D G}$ code under Gray map is linear, and we have checked that the image of our 9-class association scheme under Gray map remains to be a scheme but with different parameters. When $m>3$, the Gray map image of the $\mathcal{D G}$ code is no longer linear. It is not clear whether we can find translation schemes in elementary abelian 2-groups with the same parameters as the schemes we construct in this paper.

This paper is organized as follows. In Sect. 2, we give some preliminaries on association schemes, Galois rings, and some quaternary codes with their Lee weight distributions. In Sect. 3, we describe our construction of a 9-class association scheme coming from refining the partition of the $\mathcal{D G}$ code by its Lee weights. Its dual scheme and eigenmatrices are explicitly determined. Also a 7-class scheme as its fusion scheme and a 5-class scheme as its quotient scheme are obtained. The eigenmatrices of these schemes are listed in Appendix A. The proof of our main result is provided in Sect. 4.

\section{Preliminaries}

\subsection{Association schemes}

Let $X$ be a nonempty finite set, and a set of symmetric relations $R_{0}, R_{1}, \ldots, R_{d}$ be a partition of $X \times X$ such that $R_{0}=\{(x, x) \mid x \in X\}$. For each $i$, denote by $A_{i}$ the adjacency matrix of $R_{i}$ whose $(x, y)$ entry is 1 if $(x, y) \in R_{i}$ and 0 otherwise. We call $\left(X,\left\{R_{i}\right\}_{i=0}^{d}\right)$ a $d$-class association scheme if there exist numbers $p_{i, j}^{k}$ such that

$$
A_{i} A_{j}=\sum_{k=0}^{d} p_{i, j}^{k} A_{k}
$$


These numbers are called the intersection numbers of the scheme. The $\mathbb{C}$-linear span of $A_{0}, A_{1}, \ldots, A_{d}$ forms a semisimple algebra of dimension $d+1$, called the BoseMesner algebra of the scheme. With respect to the basis $A_{0}, A_{1}, \ldots, A_{d}$, the matrix of the multiplication by $A_{i}$ is denoted by $B_{i}$, namely

$$
A_{i}\left(A_{0}, A_{1}, \ldots, A_{d}\right)=\left(A_{0}, A_{1}, \ldots, A_{d}\right) B_{i}, \quad 0 \leq i \leq d .
$$

Since each $A_{i}$ is symmetric, this algebra is commutative. There exists a set of minimal idempotents $E_{0}, E_{1}, \ldots, E_{d}$ that also forms a basis of the algebra. The $(d+1) \times$ $(d+1)$ matrix $P$ such that

$$
\left(A_{0}, A_{1}, \ldots, A_{d}\right)=\left(E_{0}, E_{1}, \ldots, E_{d}\right) P
$$

is called the first eigenmatrix of the scheme. Dually, the $(d+1) \times(d+1)$ matrix $Q$ such that

$$
\left(E_{0}, E_{1}, \ldots, E_{d}\right)=\frac{1}{|X|}\left(A_{0}, A_{1}, \ldots, A_{d}\right) Q
$$

is called the second eigenmatrix of the scheme. Clearly, we have $P Q=|X| I$.

We call an association scheme $\left(X,\left\{R_{i}\right\}_{i=0}^{d}\right)$ a translation association scheme or a Schur ring if $X$ is an (additively written) finite abelian group and there exists a partition $S_{0}=\{0\}, S_{1}, \ldots, S_{d}$ of $X$ such that

$$
R_{i}=\left\{(x, x+y) \mid x \in X, y \in S_{i}\right\} .
$$

For brevity, we will just say that $\left(X,\left\{S_{i}\right\}_{i=0}^{d}\right)$ is an association scheme.

Assume that $\left(X,\left\{S_{i}\right\}_{i=0}^{d}\right)$ is a translation association scheme. There is an equivalence relation defined on the character group $\hat{X}$ of $X$ as follows: $\chi \sim \chi^{\prime}$ if and only if $\chi\left(S_{i}\right)=\chi^{\prime}\left(S_{i}\right)$ for each $0 \leq i \leq d$. Here $\chi(S)=\sum_{g \in S} \chi(g)$ for any $\chi \in \hat{X}$ and $S \subseteq X$. Denote by $D_{0}, D_{1}, \ldots, D_{d}$ the equivalence classes, with $D_{0}$ consisting of only the principal character. Then $\left(\hat{X},\left\{D_{i}\right\}_{i=0}^{d}\right)$ forms a translation association scheme, called the dual of $\left(X,\left\{S_{i}\right\}_{i=0}^{d}\right)$. The first eigenmatrix of the dual scheme is equal to the second eigenmatrix of the original scheme. We refer to [4] and [7] for more details.

We shall need the following well-known criterion due to Bannai [3] and Muzychuk [19], called the Bannai-Muzychuk criterion: Let $P$ be the first eigenmatrix of an association scheme $\left(X,\left\{R_{i}\right\}_{0 \leq i \leq d}\right)$, and $\Lambda_{0}:=\{0\}, \Lambda_{1}, \ldots, \Lambda_{d^{\prime}}$ be a partition of $\{0,1, \ldots, d\}$. Then $\left(X,\left\{R_{\Lambda_{i}}\right\}_{0 \leq i \leq d^{\prime}}\right)$ forms an association scheme if and only if there exists a partition $\left\{\Delta_{i}\right\}_{0 \leq i \leq d^{\prime}}$ of $\{0,1,2, \ldots, d\}$ with $\Delta_{0}=\{0\}$ such that each $\left(\Delta_{i}, \Lambda_{j}\right)$-block of $P$ has a constant row sum. Moreover, the constant row sum of the $\left(\Delta_{i}, \Lambda_{j}\right)$-block is the $(i, j)$ entry of the first eigenmatrix of the fusion scheme.

\subsection{Quaternary codes}

Let $\mu: \mathbb{Z}_{4} \rightarrow \mathbb{Z}_{2}$ denote the modulo 2 reduction map. We extend $\mu$ to $\mathbb{Z}_{4}[x]$ in the natural way. A monic polynomial $g(x) \in \mathbb{Z}_{4}[x]$ is said to be basic irreducible if $\mu(g(x))$ is a monic irreducible polynomial in $\mathbb{Z}_{2}[x]$. Let $m$ be a positive integer. 
The Galois ring $R=G R(4, m)$ of characteristic 4 with $4^{m}$ elements is defined as the quotient ring $\mathbb{Z}_{4}[x] /(f(x))$, where $f(x)$ is a monic basic irreducible polynomial of degree $m$. The collection of nonunits of $R$ forms the unique maximal ideal $2 R$, so $R$ is a local ring. Clearly, $\mu$ has a natural extension to $R[x]$, and $\mu(R)=R / 2 R$ is isomorphic to a finite field $\mathbb{F}_{q}$ of size $q=2^{m}$.

As a multiplicative group, the units $R^{*}$ of $R$ have a cyclic subgroup of order $2^{m}-1$, whose generator is denoted by $\beta$. Let $\mathcal{T}=\left\{0,1, \beta, \ldots, \beta^{2^{m}-2}\right\}$. Every element $z \in R$ can be expressed uniquely as

$$
z=A+2 B, \quad A, B \in \mathcal{T}
$$

Let $\mu(\beta)=\alpha$. Then $\alpha$ is a primitive element in $\mathbb{F}_{q}$, and $\mu(\mathcal{T})=\mathbb{F}_{q}$.

The Galois ring $R$ has a cyclic Galois group of order $m$ generated by the following Frobenius map $\sigma$ :

$$
\sigma(z)=\sigma(A+2 B)=A^{2}+2 B^{2}
$$

The trace of $z, T(z)$, from $R$ to $\mathbb{Z}_{4}$ is defined as

$$
\mathrm{T}(z)=\sum_{i=0}^{m-1} \sigma^{i}(z)
$$

and $\operatorname{tr}(x)$ is the ordinary trace function from $\mathbb{F}_{q}$ to $\mathbb{Z}_{2}$.

The Goethals code $\mathcal{G}$ of length $q=2^{m}$ over $\mathbb{Z}_{4}$ is a linear code with the following parity-check matrix

$$
H_{\mathcal{G}}=\left[\begin{array}{cccccc}
1 & 1 & 1 & 1 & \cdots & 1 \\
0 & 1 & \beta & \beta^{2} & \cdots & \beta^{2^{m}-2} \\
0 & 2 & 2 \beta^{3} & 2 \beta^{6} & \cdots & 2 \beta^{3\left(2^{m}-2\right)}
\end{array}\right]
$$

It is shown in [10, Theorem 25] that if $m$ is odd, then the Goethals code $\mathcal{G}$ has the minimum Lee distance 8 . The Delsarte-Goethals code $\mathcal{D} \mathcal{G}$ is defined as the dual of $\mathcal{G}$ over $\mathbb{Z}_{4}$, so its generator matrix is just $H_{\mathcal{G}}$.

The Delsarte-Goethals code also has the following trace description. Let $\mathbf{c}(u, a, b)$, where $u \in \mathbb{Z}_{4}, a \in R, b \in \mathcal{T}$, be a vector in $\mathbb{Z}_{4}^{q}$ indexed by the elements of $\mathcal{T}$ such that $\mathbf{c}(u, a, b)_{x}=u+\mathrm{T}\left(a x+2 b x^{3}\right)$ for all $x \in \mathcal{T}$. Then

$$
\mathcal{D G}=\left\{\mathbf{c}(u, a, b) \mid u \in \mathbb{Z}_{4}, a \in R, b \in \mathcal{T}\right\} .
$$

The Lee weight of codeword $\mathbf{c}(u, a, b)$ can be expressed as

$$
w_{L}(\mathbf{c}(u, a, b))=q-\mathfrak{R}\left(i^{u} \sum_{x \in \mathcal{T}} i^{\mathrm{T}\left(a x+2 b x^{3}\right)}\right),
$$

where $\Re$ denotes the real part of a complex number. 
Lemma 2.1 [15, Theorem 1] Let $q=2^{m}$ where $m \geq 3$ is odd. The Lee weight distribution of the Delsarte-Goethals code $\mathcal{D G}$ is

$$
B_{j}= \begin{cases}1 & \text { if } j=0 \text { or } 2 q, \\ (q-1) q(2 q-1) / 6 & \text { if } j=q \pm \sqrt{2 q}, \\ (q-1) 2 q(q+4) / 3 & \text { if } j=q \pm \sqrt{q / 2} \\ (2 q-1)\left(q^{2}-q+2\right) & \text { if } j=q .\end{cases}
$$
by

The quaternary Kerdock code $\mathcal{K}$ of length $q=2^{m}$ is a subcode of $\mathcal{D G}$ generated

$$
H_{\mathcal{K}}=\left[\begin{array}{cccccc}
1 & 1 & 1 & 1 & \cdots & 1 \\
0 & 1 & \beta & \beta^{2} & \cdots & \beta^{2^{m}-2}
\end{array}\right]
$$

Hence,

$$
\mathcal{K}=\left\{\mathbf{c}(u, a, 0) \mid u \in \mathbb{Z}_{4}, a \in R\right\} .
$$

The quaternary Preparata code $\mathcal{P}$ is a code with parity-check matrix $H_{\mathcal{K}}$. If $m$ is odd, it has the minimum Lee weight 6.

Lemma 2.2 [15, Lemma 1] Let $q=2^{m}$ where $m \geq 3$ is odd. The Lee weight distribution of the Kerdock code $\mathcal{K}$ is

$$
B_{j}= \begin{cases}1 & \text { if } j=0 \text { or } 2 q \\ 2 q(q-1) & \text { if } j=q \pm \sqrt{q / 2} \\ 4 q-2 & \text { if } j=q\end{cases}
$$

The following relations on the Kerdock code $\mathcal{K}$ will determine an abelian 4-class association scheme

$$
(x, y) \in \begin{cases}R_{0} & \text { if } w_{L}(x-y)=0 \\ R_{1} & \text { if } w_{L}(x-y)=q-\sqrt{q / 2} \\ R_{2} & \text { if } w_{L}(x-y)=q \\ R_{3} & \text { if } w_{L}(x-y)=q+\sqrt{q / 2} \\ R_{4} & \text { if } w_{L}(x-y)=2 q .\end{cases}
$$

Theorem 2.1 Let $q=2^{m}$ where $m \geq 3$ is odd. Relations (2) on the codewords of the quaternary Kerdock code define a 4-class abelian association scheme.

The above scheme is just the dual of the scheme constructed in [6, Proposition 6]. 


\section{Schemes related to the $\mathcal{D G}$ code}

It is natural to ask whether the obvious generalizations of relations (2) on the Delsarte-Goethals code $\mathcal{D G}$ will also give an abelian association scheme or not. It turns out that the answer is no. In order to get an association scheme, we should modify the relations a little:

$$
(x, y) \in \begin{cases}S_{0} & \text { if } w_{L}(x-y)=0, \\ S_{1} & \text { if } w_{L}(x-y)=q-\sqrt{2 q}, \\ S_{2} & \text { if } w_{L}(x-y)=q-\sqrt{q / 2}, \\ S_{3} & \text { if } w_{L}(x-y)=q \text { and } x-y \in \mathcal{K}, \\ S_{4} & \text { if } w_{L}(x-y)=q \text { and } x-y \notin \mathcal{K}, \\ S_{5} & \text { if } w_{L}(x-y)=q+\sqrt{q / 2}, \\ S_{6} & \text { if } w_{L}(x-y)=q+\sqrt{2 q}, \\ S_{7} & \text { if } w_{L}(x-y)=2 q .\end{cases}
$$

Theorem 3.1 Let $q=2^{m}$ where $m \geq 3$ is odd. Relations (3) on the codewords of the quaternary Delsarte-Goethals code define a 7-class abelian association scheme $\mathfrak{A}$. The first and the second eigenmatrices are given in Appendix A.

This is not the end of the story. Actually, we can provide a more refined description of relations (3) to get an abelian 9-class association scheme as follows:

$$
(x, y) \in \begin{cases}S_{0} & \text { if } w_{L}(x-y)=0, \\ S_{1} & \text { if } w_{L}(x-y)=q-\sqrt{2 q}, \\ S_{21} & \text { if } w_{L}(x-y)=q-\sqrt{q / 2} \text { and } x-y \in \mathcal{K}, \\ S_{22} & \text { if } w_{L}(x-y)=q-\sqrt{q / 2} \text { and } x-y \notin \mathcal{K}, \\ S_{3} & \text { if } w_{L}(x-y)=q \text { and } x-y \in \mathcal{K}, \\ S_{4} & \text { if } w_{L}(x-y)=q \text { and } x-y \notin \mathcal{K}, \\ S_{51} & \text { if } w_{L}(x-y)=q+\sqrt{q / 2} \text { and } x-y \notin \mathcal{K}, \\ S_{52} & \text { if } w_{L}(x-y)=q+\sqrt{q / 2} \text { and } x-y \in \mathcal{K}, \\ S_{6} & \text { if } w_{L}(x-y)=q+\sqrt{2 q}, \\ S_{7} & \text { if } w_{L}(x-y)=2 q .\end{cases}
$$

Theorem 3.2 Let $q=2^{m}$ where $m \geq 3$ is odd. Relations (4) on the codewords of the quaternary Delsarte-Goethals code define a 9-class abelian association scheme $\mathfrak{B}$. The first and the second eigenmatrices are given in Appendix A.

We leave the proofs of the above two theorems to the end of this section since they are immediate outcomes of Theorem 3.3. Recalling the trace description of the Delsarte-Goethals code $\mathcal{D G}$, we know that there is a one-to-one correspondence between the codewords of $\mathcal{D} \mathcal{G}$ and the set $\mathbb{Z}_{4} \times R \times \mathcal{T}$ given by $(u, a, b) \longleftrightarrow \mathbf{c}(u, a, b)$. 
Since $\mu(\mathcal{T})=\mathbb{F}_{q}$, there is a group isomorphism between the group $G=\mathbb{Z}_{4} \times R \times \mathbb{F}_{q}$ and the $\mathcal{D G}$ code. For $(u, a, b) \in G$, we introduce the exponential sum

$$
S(u, a, b)=\sum_{X \in \mathcal{T}} i^{u+\mathrm{T}\left(a X+2 b X^{3}\right)}+\sum_{X \in \mathcal{T}} i^{-u-\mathrm{T}\left(a X+2 b X^{3}\right)},
$$

where we have identified the element $b \in \mathbb{F}_{q}$ with its preimage $\mu^{-1}(b) \in \mathcal{T}$. Now Eq. (1) becomes

$$
w_{L}(\mathbf{c}(u, a, b))=q-S(u, a, b) / 2 .
$$

So from the weight distribution of the Delsarte-Goethals code we can see that for $(u, a, b) \in G$,

$$
S(u, a, b) \in\{ \pm 2 q, \pm 2 \sqrt{2 q}, \pm \sqrt{2 q}, 0\} .
$$

According to the value of $S(u, a, b)$, we give a partition of $G$ into ten parts as follows:

$$
\begin{aligned}
& \mathcal{R}_{0}=\{(u, a, b) \in G \mid S(u, a, b)=2 q\}=\{(0,0,0)\}, \\
& \mathcal{R}_{1}=\{(u, a, b) \in G \mid S(u, a, b)=2 \sqrt{2 q}\}, \\
& \mathcal{R}_{2}=\{(u, a, b) \in G \mid S(u, a, b)=\sqrt{2 q}, b=0\}, \\
& \mathcal{R}_{3}=\{(u, a, b) \in G \mid S(u, a, b)=\sqrt{2 q}, b \neq 0\}, \\
& \mathcal{R}_{4}=\{(u, a, b) \in G \mid S(u, a, b)=0, b=0\}, \\
& \mathcal{R}_{5}=\{(u, a, b) \in G \mid S(u, a, b)=0, b \neq 0\}, \\
& \mathcal{R}_{6}=\{(u, a, b) \in G \mid S(u, a, b)=-\sqrt{2 q}, b \neq 0\}, \\
& \mathcal{R}_{7}=\{(u, a, b) \in G \mid S(u, a, b)=-\sqrt{2 q}, b=0\}, \\
& \mathcal{R}_{8}=\{(u, a, b) \in G \mid S(u, a, b)=-2 \sqrt{2 q}\}, \quad \text { and } \\
& \mathcal{R}_{9}=\{(u, a, b) \in G \mid S(u, a, b)=-2 q\}=\{(2,0,0)\} .
\end{aligned}
$$

Since the group $G$ is abelian, its character group $\widehat{G} \cong G$. We identify an element $g=(v, c, d) \in G$ with character $\chi_{g} \in \widehat{G}$, where $\chi_{g}((u, a, b))=i^{u v+\mathrm{T}(a c+2 b d)}$ for $(u, a, b) \in G$. In order to describe the dual association scheme, we shall first provide the dual partition of the group $\widehat{G}=G$. For convenience, we will use capital letters such as $X, Y$ to denote elements in $\mathcal{T}$ and the corresponding lower cases to represent their respective projections modulo 2 in $\mathbb{F}_{q}$. Let $f_{a}(x)=x^{3}+x+a \in \mathbb{F}_{q}[x]$ and set

$$
M_{i}=\left\{a \in \mathbb{F}_{q}, a \neq 0 \mid f_{a}(x)=0 \text { has precisely } i \text { solutions in } \mathbb{F}_{q}\right\}
$$

for $i=0,1,3$. Now the dual partition is as follows:

$$
\begin{aligned}
& \mathcal{E}_{0}=\{(0,0,0)\}, \\
& \mathcal{E}_{1}=\left\{(0,0, r) \mid r \in \mathbb{F}_{q}^{*}\right\}, \\
& \mathcal{E}_{2}=\left\{\left(1, X, x^{3}\right) \mid X \in \mathcal{T}\right\} \cup\left\{\left(3,-X, x^{3}\right) \mid X \in \mathcal{T}\right\},
\end{aligned}
$$




$$
\begin{aligned}
& \mathcal{E}_{3}=\left(\left\{(1, X, r) \mid X \in \mathcal{T}, r \in \mathbb{F}_{q}\right\} \cup\left\{(3,-X, r) \mid X \in \mathcal{T}, r \in \mathbb{F}_{q}\right\}\right) \backslash \mathcal{E}_{2}, \\
& \mathcal{E}_{4}=\left.\left(0,-X+Y, x^{3}+y^{3}\right) \mid X, Y \in \mathcal{T}, X \neq Y\right\} \\
& \cup\left\{\left(2, X+Y, x^{3}+y^{3}\right) \mid X, Y \in \mathcal{T}\right\} \\
& \cup\left\{\left(2,-X-Y, x^{3}+y^{3}\right) \mid X, Y \in \mathcal{T}\right\}, \\
& \mathcal{E}_{5}=\left(\left\{(0, S, r) \mid S \in R \backslash 2 R, r \in \mathbb{F}_{q}\right\} \cup\left\{(2, S, r) \mid S \in R, r \in \mathbb{F}_{q}\right\}\right) \backslash \mathcal{E}_{4}, \\
& \mathcal{E}_{6}=\left\{\left(1, X+2 Y, x^{3}+y^{3} e\right) \mid X, Y \in \mathcal{T}, Y \neq 0, e \in \mathbb{F}_{q} \backslash M_{0}\right\} \\
& \cup\left\{\left(3,-X+2 Y, x^{3}+y^{3} e\right) \mid X, Y \in \mathcal{T}, Y \neq 0, e \in \mathbb{F}_{q} \backslash M_{0}\right\}, \\
& \mathcal{E}_{7}=\left\{\left(1, X+2 Y, x^{3}+y^{3} e\right) \mid X, Y \in \mathcal{T}, Y \neq 0, e \in M_{0}\right\} \cup\left\{\left(3,-X+2 Y, x^{3}+y^{3} e\right) \mid X, Y \in \mathcal{T}, Y \neq 0, e \in M_{0}\right\}, \\
& \mathcal{E}_{8}=\left\{\left(0,2 X, \sum_{i=1}^{4} y_{i}^{3}\right) \mid X \in \mathcal{T}^{*}, Y_{i} \in \mathcal{T}, 2 X=\sum_{i=1}^{4} Y_{i} \text { or } 2 X=Y_{1}+Y_{2}-Y_{3}-Y_{4}\right\},
\end{aligned}
$$

and

$$
\mathcal{E}_{9}=\left\{(0,2 X, r) \mid X \in \mathcal{T}^{*}, r \in \mathbb{F}_{q}\right\} \backslash \mathcal{E}_{8} .
$$

For the proof of the fact that $\mathcal{E}_{0}, \mathcal{E}_{1}, \ldots, \mathcal{E}_{9}$ form a partition of $G$, see Corollary B.2 in Appendix B.

Remark Let $m$ be an odd integer. In the study of dimensional dual hyperovals, Pasini and Yoshiara [20, Proposition 1.7], showed that the Cayley graph of the set

$$
S=\left\{\left(1, x, x^{3}\right) \mid x \in \mathbb{F}_{q}\right\} \subset \mathbb{Z}_{2} \times \mathbb{F}_{q} \times \mathbb{F}_{q}
$$

is a distance regular graph of diameter 4 . This is analogous to our set $\mathcal{E}_{2}$ above.

Theorem 3.3 Let $q=2^{m}$ where $m \geq 3$ is odd. Let $G=\mathbb{Z}_{4} \times R \times \mathbb{F}_{q}$, and define the binary relations $R_{i}=\left\{(g, h) \mid g-h \in \mathcal{R}_{i}\right\}$ for $i=0, \ldots, 9$. Then $\mathfrak{B}^{\prime}=\left(G ; R_{i}, 0 \leq\right.$ $i \leq 9)$ is a 9-class association scheme, with the first and second eigenmatrices given by $P$ and $Q$ (listed in Appendix A). The binary relations $R_{i}^{\prime}=\left\{(g, h) \mid g-h \in \mathcal{E}_{i}\right\}$ for $i=0, \ldots, 9$ define an association scheme that is dual to $\mathfrak{B}^{\prime}$, so it has the first and second eigenmatrices: $P^{\prime}=Q, Q^{\prime}=P$.

Proof Denote $s=\sqrt{2 q}$. The element of the group ring $\mathbb{C} G$ will be written as $\sum_{(u, a, b) \in G} c(u, a, b)[(u, a, b)]$, where $c(u, a, b) \in \mathbb{C}$. We set

$$
\begin{aligned}
\mathcal{N}_{2 i} & =\sum_{(u, a, b) \in G} S(u, a, b)^{i}[(u, a, b)] \text { and } \\
\mathcal{N}_{2 i+1} & =\sum_{\substack{(u, a, b) \in G \\
b=0}} S(u, a, b)^{i}[(u, a, b)]
\end{aligned}
$$


for $i=0,1, \ldots, 4$. The above transformation can be written in the matrix form as

$$
\left(\mathcal{N}_{0}, \mathcal{N}_{1}, \ldots, \mathcal{N}_{9}\right)=\left(\mathcal{R}_{0}, \mathcal{R}_{1}, \ldots, \mathcal{R}_{9}\right) T
$$

where

$$
T=\left(\begin{array}{cccccccccc}
1 & 1 & s^{2} & s^{2} & s^{4} & s^{4} & s^{6} & s^{6} & s^{8} & s^{8} \\
1 & 0 & 2 s & 0 & 4 s^{2} & 0 & 8 s^{3} & 0 & 16 s^{4} & 0 \\
1 & 1 & s & s & s^{2} & s^{2} & s^{3} & s^{3} & s^{4} & s^{4} \\
1 & 0 & s & 0 & s^{2} & 0 & s^{3} & 0 & s^{4} & 0 \\
1 & 1 & 0 & 0 & 0 & 0 & 0 & 0 & 0 & 0 \\
1 & 0 & 0 & 0 & 0 & 0 & 0 & 0 & 0 & 0 \\
1 & 0 & -s & 0 & s^{2} & 0 & -s^{3} & 0 & s^{4} & 0 \\
1 & 1 & -s & -s & s^{2} & s^{2} & -s^{3} & -s^{3} & s^{4} & s^{4} \\
1 & 0 & -2 s & 0 & 4 s^{2} & 0 & -8 s^{3} & 0 & 16 s^{4} & 0 \\
1 & 1 & -s^{2} & -s^{2} & s^{4} & s^{4} & -s^{6} & -s^{6} & s^{8} & s^{8}
\end{array}\right) .
$$

Using Maple, it is easy to compute that $\operatorname{det}(T)=-1152 s^{23}(s-1)^{2}(s+1)^{2}$, so $T$ is invertible. The key step in our proof is the completion of the following character

\begin{tabular}{|c|c|c|c|c|c|c|c|}
\hline $\mathcal{N}_{0}$ & $\mathcal{N}_{1}$ & $\mathcal{N}_{2}$ & $\mathcal{N}_{3}$ & $\mathcal{N}_{4}$ & $\mathcal{N}_{5}$ & $\mathcal{N}_{6}$ & $\mathcal{N}_{7}$ \\
\hline $\mathcal{E}_{0} / 4 q^{3}$ & $4 q^{2}$ & 0 & 0 & $8 q^{4}$ & $8 q^{3}$ & 0 & 0 \\
\hline $\mathcal{E}_{1} \quad 0$ & $4 q^{2}$ & 0 & 0 & 0 & $8 q^{3}$ & 0 & 0 \\
\hline $\mathcal{E}_{2}$ & 0 & $4 q^{3}$ & $4 q^{2}$ & 0 & 0 & $8 q^{3}(3 q-1)$ & $8 q^{2}(3 q-1)$ \\
\hline $\mathcal{E}_{3}$ & 0 & 0 & $4 q^{2}$ & 0 & 0 & 0 & $8 q^{2}(3 q-1)$ \\
\hline $\mathcal{E}_{4}$ & 0 & 0 & 0 & $8 q^{3}$ & $8 q^{2}$ & 0 & 0 \\
\hline $\mathcal{E}_{5}$ & 0 & 0 & 0 & 0 & $8 q^{2}$ & 0 & 0 \\
\hline $\mathcal{E}_{6}$ & 0 & 0 & 0 & 0 & 0 & $24 q^{3}$ & $8 q^{2}(2 q-1)$ \\
\hline $\mathcal{E}_{7}$ & 0 & 0 & 0 & 0 & 0 & 0 & $8 q^{2}(2 q-1)$ \\
\hline $\mathcal{E}_{8}$ & 0 & 0 & 0 & 0 & 0 & 0 & 0 \\
\hline $\mathcal{E}_{9}$ & 0 & 0 & 0 & 0 & 0 & 0 & 0 \\
\hline
\end{tabular}
table $\mathfrak{T}$ :

$$
\left.\begin{array}{cc}
\mathcal{N}_{8} & \mathcal{N}_{9} \\
16 q^{4}(3 q-1) & 16 q^{3}(3 q-1) \\
0 & 16 q^{3}(3 q-1) \\
0 & 0 \\
0 & 0 \\
32 q^{3}(3 q-2) & 32 q^{4} \\
32 q^{3}(q-2) & 32 q^{4} \\
0 & 0 \\
0 & 0 \\
48 q^{4} & 16 q^{3}(2 q-1) \\
0 & 16 q^{3}(2 q-1)
\end{array}\right)
$$


The next section is devoted to the computation of this table. Taking the column indexed by $\mathcal{N}_{6}$ as an illustration, we can see that

$$
g\left(\mathcal{N}_{6}\right)= \begin{cases}8 q^{3}(3 q-1) & \text { if } g \in \mathcal{E}_{2} \\ 24 q^{3} & \text { if } g \in \mathcal{E}_{6} \\ 0 & \text { otherwise }\end{cases}
$$

Thus, the character table $P$ is obtained by multiplying the above character table $\mathfrak{T}$ with the matrix $T^{-1}$ from the left. Now the assertion that $\mathfrak{B}^{\prime}=\left(G ; R_{i}, 0 \leq i \leq 9\right)$ is a 9-class association scheme is an immediate consequence of the Bannai-Muzychuk criterion $[3,19]$.

Proof of Theorems 3.1-3.2 Theorem 3.2 is clear from the statements at the beginning of this section. Theorem 3.1 follows directly from the Bannai-Muzychuk criterion and the eigenmatrices of the association scheme $\mathfrak{B}$. The scheme $\mathfrak{A}$ is a fusion scheme of $\mathfrak{B}$.

Corollary 3.1 There exists a 5-class association scheme $\mathcal{C}$ on the quotient group $G /\langle(2,0,0)\rangle$. Furthermore, there exists a 4 -class fusion scheme $\mathcal{D}$ of the scheme $\mathcal{C}$. Their first and second eigenmatrices are given in Appendix A.

Proof This can be readily checked using the eigenmatrices of the association scheme $\mathcal{B}^{\prime}$ with the help of Bannai-Muzychuk criterion.

\section{Completion of the character table $\mathfrak{T}$}

\subsection{Columns indexed by $\mathcal{N}_{0}, \mathcal{N}_{1}, \mathcal{N}_{2}$, and $\mathcal{N}_{3}$}

The first four columns of the character table $\mathfrak{T}$ can be obtained through direct computations. Here we only provide the proof of the column indexed by $\mathcal{N}_{2}$; the other three cases are similar.

For $g=(v, c, d) \in G$, we have

$$
\begin{aligned}
g\left(\mathcal{N}_{2}\right)= & \sum_{(u, a, b) \in G} S(u, a, b) i^{u v+\mathrm{T}(a c+2 b d)} \\
= & \sum_{(u, a, b) \in G} \sum_{X \in \mathcal{T}} i^{u(v+1)+\mathrm{T}(a(c+X))+2 \mathrm{~T}\left(b\left(d+X^{3}\right)\right)} \\
& +\sum_{(u, a, b) \in G} \sum_{X \in \mathcal{T}} i^{u(v-1)+\mathrm{T}(a(c-X))+2 \mathrm{~T}\left(b\left(d-X^{3}\right)\right)}
\end{aligned}
$$




$$
\begin{aligned}
& =\sum_{X \in \mathcal{T}} \sum_{u \in \mathbb{Z}_{4}} i^{u(v+1)} \sum_{a \in R} i^{\mathrm{T}(a(c+X))} \sum_{b \in \mathcal{T}} i^{2 \mathrm{~T}\left(b\left(d+X^{3}\right)\right)} \\
& +\sum_{X \in \mathcal{T}} \sum_{u \in \mathbb{Z}_{4}} i^{u(v-1)} \sum_{a \in R} i^{\mathrm{T}(a(c-X))} \sum_{b \in \mathcal{T}} i^{2 \mathrm{~T}\left(b\left(d-X^{3}\right)\right)} \\
& = \begin{cases}4 q^{3} & \text { if } g \in \mathcal{E}_{2}, \\
0 & \text { otherwise, }\end{cases}
\end{aligned}
$$

and this completes our proof.

4.2 Columns indexed by $\mathcal{N}_{4}, \mathcal{N}_{5}, \mathcal{N}_{6}, \mathcal{N}_{7}$, and $\mathcal{N}_{9}$

First, a direct calculation gives that

$$
\begin{aligned}
g\left(\mathcal{N}_{4}\right)= & \sum_{(u, a, b) \in G} S(u, a, b)^{2} i^{u v+\mathrm{T}(a c+2 b d)} \\
= & \sum_{u \in \mathbb{Z}_{4}} i^{u(v+2)} \sum_{X, Y \in \mathcal{T}} \sum_{a \in R} i^{\mathrm{T}(a(c+X+Y))} \sum_{b \in \mathbb{F}_{q}} i^{\mathrm{T}\left(2 b\left(d+X^{3}+Y^{3}\right)\right)} \\
& +\sum_{u \in \mathbb{Z}_{4}} i^{u(v-2)} \sum_{X, Y \in \mathcal{T}} \sum_{a \in R} i^{\mathrm{T}(a(c-X-Y))} \sum_{b \in \mathbb{F}_{q}} i^{\mathrm{T}\left(2 b\left(d-X^{3}-Y^{3}\right)\right)} \\
& +2 \sum_{u \in \mathbb{Z}_{4}} i^{u v} \sum_{X, Y \in \mathcal{T}} \sum_{a \in R} i^{\mathrm{T}(a(c+X-Y))} \sum_{b \in \mathbb{F}_{q}} i^{\mathrm{T}\left(2 b\left(d+X^{3}-Y^{3}\right)\right)}
\end{aligned}
$$

for $g=(v, c, d) \in G$.

Suppose that $v=0$ and $c=-Z+W$ for some $Z, W \in \mathcal{T}$ with $Z \neq W$. Then only the last term in the above sum possibly contributes. Using (b) of Lemma B.2, we see that it will contribute $8 q^{3}$ if $d=z^{3}+w^{3}$ and zero otherwise. A routine analysis shows that

$$
g\left(\mathcal{N}_{4}\right)= \begin{cases}8 q^{4} & \text { if } g \in \mathcal{E}_{0} \\ 8 q^{3} & \text { if } g \in \mathcal{E}_{4} \\ 0 & \text { otherwise }\end{cases}
$$

and this completes the column indexed by $\mathcal{N}_{4}$.

The column indexed by $\mathcal{N}_{5}$ can be checked exactly as same as $\mathcal{N}_{4}$.

Now we continue to treat the column $\mathcal{N}_{6}$ :

$$
\begin{aligned}
g\left(\mathcal{N}_{6}\right) & =\sum_{(u, a, b) \in G} S(u, a, b)^{3} i^{u v+\mathrm{T}(a c+2 b d)} \\
& =\sum_{u \in \mathbb{Z}_{4}} i^{u(v+3)} \sum_{X, Y, Z \in \mathcal{T}} \sum_{a \in R} i^{\mathrm{T}(a(c+X+Y+Z))} \sum_{b \in \mathbb{F}_{q}} i^{\mathrm{T}\left(2 b\left(d+X^{3}+Y^{3}+Z^{3}\right)\right)}
\end{aligned}
$$




$$
\begin{aligned}
& +\sum_{u \in \mathbb{Z}_{4}} i^{u(v-3)} \sum_{X, Y, Z \in \mathcal{T}} \sum_{a \in R} i^{\mathrm{T}(a(c-X-Y-Z))} \sum_{b \in \mathbb{F}_{q}} i^{\mathrm{T}\left(2 b\left(d-X^{3}-Y^{3}-Z^{3}\right)\right)} \\
& +3 \sum_{u \in \mathbb{Z}_{4}} i^{u(v+1)} \sum_{X, Y, Z \in \mathcal{T}} \sum_{a \in R} i^{\mathrm{T}(a(c+X+Y-Z))} \sum_{b \in \mathbb{F}_{q}} i^{\mathrm{T}\left(2 b\left(d+X^{3}+Y^{3}-Z^{3}\right)\right)} \\
& +3 \sum_{u \in \mathbb{Z}_{4}} i^{u(v-1)} \sum_{X, Y, Z \in \mathcal{T}} \sum_{a \in R} i^{\mathrm{T}(a(c-X-Y+Z))} \sum_{b \in \mathbb{F}_{q}} i^{\mathrm{T}\left(2 b\left(d-X^{3}-Y^{3}+Z^{3}\right)\right)}
\end{aligned}
$$

for $g=(v, c, d) \in G$.

By the definition of the sets $\mathcal{E}_{0}, \mathcal{E}_{1}, \ldots, \mathcal{E}_{9}$ we can verify that the value $g\left(\mathcal{N}_{6}\right)$ vanishes if $g \notin \mathcal{E}_{2} \cup \mathcal{E}_{6}$. Then we only need to deal with the case where $g \in \mathcal{E}_{2} \cup \mathcal{E}_{6}$. First, suppose that $g=(v, c, d)=\left(1, W, w^{3}\right) \in \mathcal{E}_{2}$ for some $W \in \mathcal{T}$. Then only the first and last terms in the above sum will contribute. Using (d) of Lemma B.2, we see that the first term will contribute $4 q^{3}$ if $d=w^{3}$ and zero otherwise. A similar analysis shows that the last term will contribute $12 q^{3}(2 q-1)$ if $d=w^{3}$ and zero otherwise by (c) of Lemma B.2. The analysis for $g=\left(3,-W, w^{3}\right) \in \mathcal{E}_{2}$ is similar. So $g\left(\mathcal{N}_{6}\right)=8 q^{3}(3 q-1)$ for $g \in \mathcal{E}_{2}$. Secondly, suppose that $g=(v, c, d) \in \mathcal{E}_{6}$. The statement can be proved similarly using Lemmas B.7-B.8 in Appendix B.

We come to treat the column $\mathcal{N}_{7}$ :

$$
\begin{aligned}
g\left(\mathcal{N}_{7}\right)= & \sum_{\substack{(u, a, b) \in G \\
b=0}} S(u, a, b)^{3} i^{u v+\mathrm{T}(a c+2 b d)} \\
= & \sum_{u \in \mathbb{Z}_{4}} i^{u(v+3)} \sum_{X, Y, Z \in \mathcal{T}} \sum_{a \in R} i^{\mathrm{T}(a(c+X+Y+Z))} \\
& +\sum_{u \in \mathbb{Z}_{4}} i^{u(v-3)} \sum_{X, Y, Z \in \mathcal{T}} \sum_{a \in R} i^{\mathrm{T}(a(c-X-Y-Z))} \\
& +3 \sum_{u \in \mathbb{Z}_{4}} i^{u(v+1)} \sum_{X, Y, Z \in \mathcal{T}} \sum_{a \in R} i^{\mathrm{T}(a(c+X+Y-Z))} \\
& +3 \sum_{u \in \mathbb{Z}_{4}} i^{u(v-1)} \sum_{X, Y, Z \in \mathcal{T}} \sum_{a \in R} i^{\mathrm{T}(a(c-X-Y+Z))}
\end{aligned}
$$

for $g=(v, c, d) \in G$. The remaining discussion is similar as for the column $\mathcal{N}_{6}$ by using Lemma B.3.

At last we come to treat the column $\mathcal{N}_{9}$ :

$$
\begin{aligned}
g\left(\mathcal{N}_{9}\right)= & \sum_{\substack{(u, a, b) \in G \\
b=0}} S(u, a, b)^{4} i^{u v+\mathrm{T}(a c+2 b d)} \\
= & \sum_{u \in \mathbb{Z}_{4}} i^{u v} \sum_{X, Y, Z, W \in \mathcal{T}} \sum_{a \in R} i^{\mathrm{T}(a(c+X+Y+Z+W))} \\
& +\sum_{u \in \mathbb{Z}_{4}} i^{u v} \sum_{X, Y, Z, W \in \mathcal{T}} \sum_{a \in R} i^{\mathrm{T}(a(c-X-Y-Z-W))}
\end{aligned}
$$




$$
\begin{aligned}
& +4 \sum_{u \in \mathbb{Z}_{4}} i^{u(v+2)} \sum_{X, Y, Z, W \in \mathcal{T}} \sum_{a \in R} i^{\mathrm{T}(a(c+X+Y+Z-W))} \\
& +4 \sum_{u \in \mathbb{Z}_{4}} i^{u(v-2)} \sum_{X, Y, Z, W \in \mathcal{T}} \sum_{a \in R} i^{\mathrm{T}(a(c-X-Y-Z+W))} \\
& +3 \sum_{u \in \mathbb{Z}_{4}} i^{u v} \sum_{X, Y, Z, W \in \mathcal{T}} \sum_{a \in R} i^{\mathrm{T}(a(c+X+Y-Z-W))} \\
& +3 \sum_{u \in \mathbb{Z}_{4}} i^{u v} \sum_{X, Y, Z, W \in \mathcal{T}} \sum_{a \in R} i^{\mathrm{T}(a(c-X-Y+Z+W))}
\end{aligned}
$$

for $g=(v, c, d) \in G$. The remaining discussion is similar as for the column $\mathcal{N}_{6}$ by using Corollary B.1.

\subsection{Column indexed by $\mathcal{N}_{8}$}

This is the most difficult case. When $g=(0,0,0)$, the identity $g\left(\mathcal{N}_{8}\right)=16 q^{4}(3 q-1)$ is direct. When $g \in \mathcal{E}_{8}$, we can prove that $g\left(\mathcal{N}_{8}\right)=48 q^{4}$ by Corollaries B.3-B.4. The exponential sum $\xi(a, b)=\sum_{X \in \mathcal{T}} i^{\mathrm{T}\left(a X+2 b X^{3}\right)}$ is closely related to $S(u, a, b)$. We further introduce two exponential sums

$$
\mathbf{E}(c, d):=\sum_{a \in R} \sum_{b \in \mathbb{F}_{q}}\left(\xi^{4}(a, b)+\overline{\xi^{4}(a, b)}+6 \xi^{2}(a, b) \overline{\xi^{2}(a, b)}\right) i^{\mathrm{T}(a c+2 b d)}
$$

and

$$
\mathbf{F}(c, d):=\sum_{a \in R} \sum_{b \in \mathbb{F}_{q}}\left(\xi^{3}(a, b) \overline{\xi(a, b)}+\xi(a, b) \overline{\xi^{3}(a, b)}\right) i^{u v+\mathrm{T}(a c+2 b d)} .
$$

Then

$$
\begin{aligned}
g\left(\mathcal{N}_{8}\right) & =\sum_{(u, a, b) \in G} S(u, a, b)^{4} i^{u v+\mathrm{T}(a c+2 b d)} \\
& =\sum_{u \in \mathbb{Z}_{4}} i^{u v} \mathbf{E}(c, d)+4 \sum_{u \in \mathbb{Z}_{4}} i^{u(v+2)} \mathbf{F}(c, d) .
\end{aligned}
$$

So it is enough to determine the distribution of $\mathbf{E}(c, d)$ and $\mathbf{F}(c, d)$. Since the determination is very technical and complex, we prefer to leave it in Appendix B. Using Lemmas C.2-C.4, it is now a routine check to see that

$$
g\left(\mathcal{N}_{8}\right)= \begin{cases}2^{3 m+6}\left(3 \cdot 2^{m-1}-1\right) & \text { if } g \in \mathcal{E}_{4}, \\ 2^{3 m+6}\left(2^{m-1}-1\right) & \text { if } g \in \mathcal{E}_{5} .\end{cases}
$$

\section{Conclusion}

In this paper, we construct a 9-class scheme from a refinement of the partition of the $\mathcal{D G}$ code by its Lee weights. We get explicit expressions of the dual scheme 
of this 9-class scheme by manipulations of complicated exponential sums and heavy computations. There is an interesting "nonsymmetry" between the characterization of the 9-class scheme and its dual in the sense that the description of the original scheme reflects the properties of the underlying code, while we see nothing about the code in the description of the dual scheme. Moreover, the dual scheme cannot be described by the cosets of the Goethals codes as far as we see it. It will be interesting to see what code properties are reflected in the dual scheme.

Bonnecaze and Duursma [6] showed that the partition of the Kerdock (resp. shortened Kerdock) code induced by the complete weight enumerators gives rise to an association scheme. Using this scheme and the complete weight enumerator of the Kerdock (resp. shortened Kerdock) code, they also showed that the complete weight enumerator of each coset of the dual code, namely Preparata (resp. punctured Preparata) code, can be explicitly determined. Thus, it is reasonable to believe that this is also true for the $\mathcal{D G}$ code, which would give rise to a 22-class association scheme. Since the complete weight enumerator of the $\mathcal{D G}$ code has been explicitly determined by Shin, Kumar, and Helleseth [21], once we figure out the parameters of this conjectured scheme, then theoretically we know all about the complete weight enumerators of each coset of the Goethals code. The coset weight enumerators of the Goethals code have been studied by Helleseth and Zinoviev [13, 14]. We mention that our 9-class scheme is not a fusion scheme of this conjectured 22-class scheme.

Davis and Xiang [8] constructed the first known examples where the nonhomomorphic bijection approach outlined by Hagita and Schmidt [9] can produce negative Latin square-type partial difference sets in groups that previously had no known constructions. The Cayley graphs of partial difference sets are strongly regular and so yield two-class association schemes. Therefore, it is interesting to investigate whether there are translation schemes over elementary abelian 2-groups with the same parameters as those constructed in $[1,6]$ and this paper from the various $\mathbb{Z}_{4}$-linear codes. When $m=3$, the Gray map image of the $\mathcal{D G}$ code is a $\mathbb{Z}_{2}$-linear code, and we checked that the Gray map image of the 9-class scheme remains a scheme but with different parameters. However, when $m>3$, the binary $\mathcal{D} \mathcal{G}$ code is no longer linear, and no scheme arises naturally in this way.

Acknowledgements The authors thank the two anonymous referees for their constructive comments and suggestions that greatly improved the readability of this article. S. Hu was supported by the Scholarship Award for Excellent Doctoral Student granted by Ministry of Education. T. Feng was supported in part by Fundamental Research Fund for the Central Universities of China, Zhejiang Provincial Natural Science Foundation under Grant No. LQ12A01019, in part by the National Natural Science Foundation of China under Grant No. 11201418 and in part by the Research Fund for Doctoral Programs from the Ministry of Education of China under Grant No. 20120101120089. G. Ge was supported by the National Natural Science Foundation of China under Grant No. 61171198 and Zhejiang Provincial Natural Science Foundation of China under Grant No. LZ13A010001.

\section{Appendix A}

Let $q=2^{m}$ where $m \geq 3$ is odd. Denote $s=\sqrt{2 q}$. 
The first and second eigenmatrices of the association scheme $\mathfrak{A}$ :

$$
\begin{aligned}
& \begin{array}{llll}
\mathcal{R}_{0} & \mathcal{R}_{1} & \mathcal{R}_{2} \cup \mathcal{R}_{3} & \mathcal{R}_{4}
\end{array} \\
& \mathcal{E}_{0} \quad\left(\begin{array}{cccc}
1 & 1 / 24 s^{6}-1 / 8 s^{4}+1 / 12 s^{2} & 1 / 2 s^{4}-4 / 3 s^{2}+1 / 12 s^{6} & -2+2 s^{2} \\
1 & -1 / 12 s^{4}+1 / 12 s^{2} & 1 / 3 s^{4}-4 / 3 s^{2} & -2+2 s^{2}
\end{array}\right. \\
& \mathcal{E}_{2} \quad 1 \quad 1 / 12 s^{5}-1 / 4 s^{3}+1 / 6 s \quad 1 / 2 s^{3}-4 / 3 s+1 / 12 s^{5} \quad 0 \\
& P=\begin{array}{c|cccc}
\mathcal{E}_{3} \cup \mathcal{E}_{7} & 1 & -1 / 6 s^{3}+1 / 6 s & 1 / 3 s^{3}-4 / 3 s & 0 \\
\mathcal{E}_{4} & 1 & 1 / 8 s^{4}-1 / 4 s^{2} & 0
\end{array} \\
& \begin{array}{l|llll}
\mathcal{E}_{5} & 1 & -1 / 4 s^{2} & 0 & -2 \\
1 & 1 / 12 s^{3}+1 / 6 s & 0 & -2
\end{array} \\
& \begin{array}{c|cccc}
\mathcal{E}_{6} & 1 & 1 / 12 s^{3}+1 / 6 s & -4 / 3 s-1 / 6 s^{3} & 0 \\
\mathcal{E}_{8} & 1 & 1 / 24 s^{4}+1 / 12 s^{2} & -4 / 3 s^{2}-1 / 6 s^{4} & -2+2 s^{2}
\end{array} \\
& \mathcal{R}_{5} \quad \mathcal{R}_{6} \cup \mathcal{R}_{7} \\
& 1 / 4 s^{6}-3 / 4 s^{4}+1 / 2 s^{2} \quad 1 / 2 s^{4}-4 / 3 s^{2}+1 / 12 s^{6} \\
& -1 / 2 s^{4}+1 / 2 s^{2} \quad 1 / 3 s^{4}-4 / 3 s^{2} \\
& 0-1 / 12 s^{5}+4 / 3 s-1 / 2 s^{3} \\
& 0-1 / 3 s^{3}+4 / 3 s \\
& -1 / 4 s^{4}+1 / 2 s^{2} \quad 0 \\
& 1 / 2 s^{2} \quad 0 \\
& 0 \quad 1 / 6 s^{3}+4 / 3 s \\
& 1 / 4 s^{4}+1 / 2 s^{2} \quad-4 / 3 s^{2}-1 / 6 s^{4} \\
& \mathcal{R}_{8} \quad \mathcal{R}_{9} \\
& \begin{array}{cc}
1 / 24 s^{6}-1 / 8 s^{4}+1 / 12 s^{2} & 1 \\
-1 / 12 s^{4}+1 / 12 s^{2} & 1
\end{array} \\
& -1 / 12 s^{5}+1 / 4 s^{3}-1 / 6 s-1 \\
& 1 / 6 s^{3}-1 / 6 s-1 \\
& 1 / 8 s^{4}-1 / 4 s^{2} \quad 1 \\
& -1 / 4 s^{2} \quad 1 \\
& \left.\begin{array}{cc}
-1 / 12 s^{3}-1 / 6 s & -1 \\
1 / 24 s^{4}+1 / 12 s^{2} & 1
\end{array}\right)
\end{aligned}
$$$$
Q=\left(\begin{array}{ccccc}
1 & 1 / 2 s^{2}-1 & s^{2} & 1 / 2\left(s^{2}-2\right) s^{2} & 1 / 2 s^{2}\left(s^{2}-1\right) \\
1 & -1 & 2 s & -2 s & 3 / 2 s^{2} \\
1 & 1 / 2 s^{2}-1 & s & 1 / 2\left(s^{2}-2\right) s & 0 \\
1 & -1 & s & -s & 0 \\
1 & 1 / 2 s^{2}-1 & 0 & 0 & -1 / 2 s^{2} \\
1 & -1 & 0 & 0 & -1 / 2 s^{2} \\
1 & -1 & -s & s & 0 \\
1 & 1 / 2 s^{2}-1 & -s & -1 / 2\left(s^{2}-2\right) s & 0
\end{array}\right.
$$ 


$$
\left.\begin{array}{ccc}
1 / 4 s^{2}\left(s^{4}-3 s^{2}+2\right) & 1 / 6 s^{2}\left(s^{4}-3 s^{2}+2\right) & 1 / 6 s^{4}-1 / 2 s^{2}+1 / 3 \\
-3 / 2 s^{2} & 1 / 3 s\left(s^{2}+2\right) & 1 / 6 s^{2}+1 / 3 \\
0 & -1 / 3 s\left(s^{2}-1\right) & 1 / 3-1 / 3 s^{2} \\
-1 / 4\left(s^{2}-2\right) s^{2} & 0 & 1 / 6 s^{4}-1 / 2 s^{2}+1 / 3 \\
1 / 2 s^{2} & 0 & 1 / 6 s^{2}+1 / 3 \\
0 & 1 / 3 s\left(s^{2}-1\right) & 1 / 3-1 / 3 s^{2} \\
-3 / 2 s^{2} & -1 / 3 s\left(s^{2}+2\right) & 1 / 6 s^{2}+1 / 3 \\
1 / 4 s^{2}\left(s^{4}-3 s^{2}+2\right) & -1 / 6 s^{2}\left(s^{4}-3 s^{2}+2\right) & 1 / 6 s^{4}-1 / 2 s^{2}+1 / 3
\end{array}\right)
$$

The first and second eigenmatrices of the association schemes $\mathfrak{B}$ and $\mathfrak{B}^{\prime}$ :

$$
\begin{aligned}
& \begin{array}{llll}
\mathcal{R}_{0} & \mathcal{R}_{1} & \mathcal{R}_{2} & \mathcal{R}_{3}
\end{array}
\end{aligned}
$$

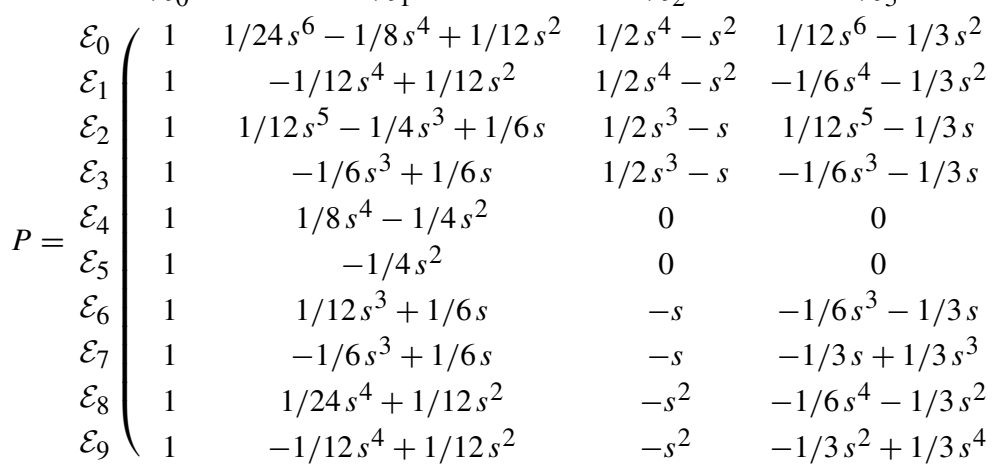

$$
\begin{aligned}
& \begin{array}{cccc}
\mathcal{R}_{4} & \mathcal{R}_{5} & \mathcal{R}_{6} & \mathcal{R}_{7} \\
-2+2 s^{2} & 1 / 4 s^{6}-3 / 4 s^{4}+1 / 2 s^{2} & 1 / 12 s^{6}-1 / 3 s^{2} & 1 / 2 s^{4}-s^{2}
\end{array} \\
& -2+2 s^{2} \quad-1 / 2 s^{4}+1 / 2 s^{2} \quad-1 / 6 s^{4}-1 / 3 s^{2} \quad 1 / 2 s^{4}-s^{2} \\
& 0 \quad 0 \quad-1 / 12 s^{5}+1 / 3 s-1 / 2 s^{3}+s \\
& \begin{array}{lccc}
0 & 0 & 1 / 6 s^{3}+1 / 3 s & -1 / 2 s^{3}+s
\end{array} \\
& \begin{array}{cccc}
-2 & 1 / 2 s^{2} & 0 & 0
\end{array} \\
& 0 \quad 0 \quad 1 / 6 s^{3}+1 / 3 s \quad s \\
& 0 \quad 0 \quad 1 / 3 s-1 / 3 s^{3} \quad s \\
& \begin{array}{cccc}
-2+2 s^{2} & 1 / 4 s^{4}+1 / 2 s^{2} & -1 / 6 s^{4}-1 / 3 s^{2} & -s^{2} \\
-2+2 s^{2} & -1 / 2 s^{4}+1 / 2 s^{2} & -1 / 3 s^{2}+1 / 3 s^{4} & -s^{2}
\end{array} \\
& \left.\begin{array}{cc}
\mathcal{R}_{8} & \mathcal{R}_{9} \\
1 / 24 s^{6}-1 / 8 s^{4}+1 / 12 s^{2} & 1 \\
-1 / 12 s^{4}+1 / 12 s^{2} & 1 \\
-1 / 12 s^{5}+1 / 4 s^{3}-1 / 6 s & -1 \\
1 / 6 s^{3}-1 / 6 s & -1 \\
1 / 8 s^{4}-1 / 4 s^{2} & 1 \\
-1 / 4 s^{2} & 1 \\
-1 / 12 s^{3}-1 / 6 s & -1 \\
1 / 6 s^{3}-1 / 6 s & -1 \\
1 / 24 s^{4}+1 / 12 s^{2} & 1 \\
-1 / 12 s^{4}+1 / 12 s^{2} & 1
\end{array}\right)
\end{aligned}
$$




$$
\begin{aligned}
& Q=\left(\begin{array}{cccccc}
1 & 1 / 2 s^{2}-1 & s^{2} & 1 / 2\left(s^{2}-2\right) s^{2} & 1 / 2 s^{2}\left(s^{2}-1\right) & 1 / 4 s^{2}\left(s^{4}-3 s^{2}+2\right) \\
1 & -1 & 2 s & -2 s & 3 / 2 s^{2} & -3 / 2 s^{2} \\
1 & 1 / 2 s^{2}-1 & s & 1 / 2\left(s^{2}-2\right) s & 0 & 0 \\
1 & -1 & s & -s & 0 & 0 \\
1 & 1 / 2 s^{2}-1 & 0 & 0 & -1 / 2 s^{2} & -1 / 4\left(s^{2}-2\right) s^{2} \\
1 & -1 & 0 & 0 & -1 / 2 s^{2} & 1 / 2 s^{2} \\
1 & -1 & -s & s & 0 & 0 \\
1 & 1 / 2 s^{2}-1 & -s & -1 / 2\left(s^{2}-2\right) s & 0 & 0 \\
1 & -1 & -2 s & 2 s & 3 / 2 s^{2} & -3 / 2 s^{2} \\
1 & 1 / 2 s^{2}-1 & -s^{2} & -1 / 2\left(s^{2}-2\right) s^{2} & 1 / 2 s^{2}\left(s^{2}-1\right) & 1 / 4 s^{2}\left(s^{4}-3 s^{2}+2\right)
\end{array}\right. \\
& \left.\begin{array}{cccc}
1 / 6 s^{2}\left(s^{4}-3 s^{2}+2\right) & 1 / 12 s^{2}\left(s^{4}-4\right) & 1 / 6 s^{4}-1 / 2 s^{2}+1 / 3 & 1 / 12 s^{4}-1 / 3 \\
1 / 3 s\left(s^{2}+2\right) & -1 / 3 s\left(s^{2}+2\right) & 1 / 6 s^{2}+1 / 3 & -1 / 6 s^{2}-1 / 3 \\
-1 / 3 s\left(s^{2}-1\right) & -1 / 6 s\left(s^{2}+2\right) & 1 / 3-1 / 3 s^{2} & -1 / 6 s^{2}-1 / 3 \\
-1 / 3 s\left(s^{2}-1\right) & 1 / 3 s\left(s^{2}-1\right) & 1 / 3-1 / 3 s^{2} & -1 / 3+1 / 3 s^{2} \\
0 & 0 & 1 / 6 s^{4}-1 / 2 s^{2}+1 / 3 & 1 / 12 s^{4}-1 / 3 \\
0 & 0 & 1 / 6 s^{2}+1 / 3 & -1 / 6 s^{2}-1 / 3 \\
1 / 3 s\left(s^{2}-1\right) & -1 / 3 s\left(s^{2}-1\right) & 1 / 3-1 / 3 s^{2} & -1 / 3+1 / 3 s^{2} \\
1 / 3 s\left(s^{2}-1\right) & 1 / 6 s\left(s^{2}+2\right) & 1 / 3-1 / 3 s^{2} & -1 / 6 s^{2}-1 / 3 \\
-1 / 3 s\left(s^{2}+2\right) & 1 / 3 s\left(s^{2}+2\right) & 1 / 6 s^{2}+1 / 3 & -1 / 6 s^{2}-1 / 3 \\
-1 / 6 s^{2}\left(s^{4}-3 s^{2}+2\right) & -1 / 12 s^{2}\left(s^{4}-4\right) & 1 / 6 s^{4}-1 / 2 s^{2}+1 / 3 & 1 / 12 s^{4}-1 / 3
\end{array}\right) .
\end{aligned}
$$

The first and second eigenmatrices of the association scheme $\mathcal{C}$ :

$$
\begin{aligned}
& P=\left(\begin{array}{cccc}
1 & 1 / 24 s^{6}-1 / 8 s^{4}+1 / 12 s^{2} & 1 / 2 s^{4}-s^{2} & 1 / 12 s^{6}-1 / 3 s^{2} \\
1 & -1 / 12 s^{4}+1 / 12 s^{2} & 1 / 2 s^{4}-s^{2} & -1 / 6 s^{4}-1 / 3 s^{2} \\
1 & 1 / 8 s^{4}-1 / 4 s^{2} & 0 & 0 \\
1 & -1 / 4 s^{2} & 0 & 0 \\
1 & 1 / 24 s^{4}+1 / 12 s^{2} & -s^{2} & -1 / 6 s^{4}-1 / 3 s^{2} \\
1 & -1 / 12 s^{4}+1 / 12 s^{2} & -s^{2} & -1 / 3 s^{2}+1 / 3 s^{4}
\end{array}\right. \\
& \left.\begin{array}{cc}
s^{2}-1 & 1 / 8 s^{6}-3 / 8 s^{4}+1 / 4 s^{2} \\
s^{2}-1 & -1 / 4 s^{4}+1 / 4 s^{2} \\
-1 & -1 / 8 s^{4}+1 / 4 s^{2} \\
-1 & 1 / 4 s^{2} \\
s^{2}-1 & 1 / 8 s^{4}+1 / 4 s^{2} \\
s^{2}-1 & -1 / 4 s^{4}+1 / 4 s^{2}
\end{array}\right),
\end{aligned}
$$




$$
\begin{gathered}
Q=\left(\begin{array}{cccc}
1 & 1 / 2 s^{2}-1 & 1 / 2 s^{2}\left(s^{2}-1\right) & 1 / 4 s^{2}\left(s^{4}-3 s^{2}+2\right) \\
1 & -1 & 3 / 2 s^{2} & -3 / 2 s^{2} \\
1 & 1 / 2 s^{2}-1 & 0 & 0 \\
1 & -1 & 0 & 0 \\
1 & 1 / 2 s^{2}-1 & -1 / 2 s^{2} & -1 / 4\left(s^{2}-2\right) s^{2} \\
1 & -1 & -1 / 2 s^{2} & 1 / 2 s^{2} \\
1 / 6 s^{4}-1 / 2 s^{2}+1 / 3 & 1 / 12 s^{4}-1 / 3 \\
1 / 6 s^{2}+1 / 3 & -1 / 6 s^{2}-1 / 3 \\
1 / 3-1 / 3 s^{2} & -1 / 6 s^{2}-1 / 3 \\
1 / 3-1 / 3 s^{2} & -1 / 3+1 / 3 s^{2} \\
4 & 1 / 6 s^{2}+1 / 3 & -1 / 6 s^{2}-1 / 3
\end{array}\right)
\end{gathered}
$$

The first and second eigenmatrices of the association scheme $\mathcal{D}$ :

$$
\begin{aligned}
& P=\left(\begin{array}{ccc}
1 & 1 / 24 s^{6}-1 / 8 s^{4}+1 / 12 s^{2} & 1 / 2 s^{4}-4 / 3 s^{2}+1 / 12 s^{6} \\
1 & -1 / 12 s^{4}+1 / 12 s^{2} & 1 / 3 s^{4}-4 / 3 s^{2} \\
1 & 1 / 8 s^{4}-1 / 4 s^{2} & 0 \\
1 & -1 / 4 s^{2} & 0 \\
1 & 1 / 24 s^{4}+1 / 12 s^{2} & -4 / 3 s^{2}-1 / 6 s^{4}
\end{array}\right. \\
& s^{2}-1 \quad 1 / 8 s^{6}-3 / 8 s^{4}+1 / 4 s^{2} \\
& s^{2}-1 \quad-1 / 4 s^{4}+1 / 4 s^{2} \\
& -1 \quad-1 / 8 s^{4}+1 / 4 s^{2} \\
& -1 \quad 1 / 4 s^{2} \\
& \left.s^{2}-1 \quad 1 / 8 s^{4}+1 / 4 s^{2}\right) \\
& Q=\left(\begin{array}{ccc}
1 & 1 / 2 s^{2}-4 / 3+1 / 12 s^{4} & 1 / 2 s^{2}\left(s^{2}-1\right) \\
1 & -4 / 3-1 / 6 s^{2} & 3 / 2 s^{2} \\
1 & 1 / 3 s^{2}-4 / 3 & 0 \\
1 & 1 / 2 s^{2}-4 / 3+1 / 12 s^{4} & -1 / 2 s^{2} \\
1 & -4 / 3-1 / 6 s^{2} & -1 / 2 s^{2}
\end{array}\right. \\
& \left.\begin{array}{cc}
1 / 4 s^{2}\left(s^{4}-3 s^{2}+2\right) & 1 / 6 s^{4}-1 / 2 s^{2}+1 / 3 \\
-3 / 2 s^{2} & 1 / 6 s^{2}+1 / 3 \\
0 & 1 / 3-1 / 3 s^{2} \\
-1 / 4\left(s^{2}-2\right) s^{2} & 1 / 6 s^{4}-1 / 2 s^{2}+1 / 3 \\
1 / 2 s^{2} & 1 / 6 s^{2}+1 / 3
\end{array}\right) .
\end{aligned}
$$




\section{Appendix B}

Let $<$ be any ordering of the elements of $\mathcal{T}$.

Lemma B.1 [12, Lemma 2] Let $\mathbf{e}=\left(e_{X}\right)_{X \in \mathcal{T}}$, and let $E_{j}=\left\{X \mid e_{X}=j\right\}$ for $j=$ $0,1,2,3$. The equation given by

$$
\sum_{X \in \mathcal{T}} e_{X} X=A+2 B, \quad A, B \in \mathcal{T}, e_{X} \in \mathbb{Z}_{4},
$$

is equivalent to the two binary equations

$$
a=\sum_{X \in E_{1} \cup E_{3}} x
$$

and

$$
b^{2}=\sum_{X \in E_{2} \cup E_{3}} x^{2}+\sum_{\substack{X, Y \in E_{1} \cup E_{3} \\ X<Y}} x y .
$$

We set $2 R=\{2 x \mid x \in R\}$ and $-\mathcal{T}=\{-X \mid X \in \mathcal{T}\}$.

Lemma B.2 [6, Theorem 1] Let $R=G R(4, m), m>0$, and $\mathcal{T}$ be the Teichmuller set.

(a) The multiset $\mathcal{T}+2 \mathcal{T}=\{X+2 Y \mid X, Y \in \mathcal{T}\}$ contains each element of $R$ with multiplicity one.

(b) The multiset $\mathcal{T}-\mathcal{T}=\{X-Y \mid X, Y \in \mathcal{T}\}$ contains 0 with multiplicity $2^{m}$, no other elements of $2 R$, and the elements outside $2 R$ with multiplicity one.

(c) The multiset $\mathcal{T}+\mathcal{T}=\{X+Y \mid X, Y \in \mathcal{T}\}$ contains the elements of $2 R$ with multiplicity one and half of the elements outside $2 R$ with multiplicity two.

(d) The multiset $\mathcal{T}+\mathcal{T}$ and $-(\mathcal{T}+\mathcal{T})$ coincide for $m$ even. For $m$ odd, they intersect in $2 R$. In particular, elements of $-\mathcal{T}$ occur in $\mathcal{T}+\mathcal{T}$ only for $m$ even.

The next two results are natural generalizations of Lemma B.2.

Lemma B.3 Let $R=G R(4, m), m$ odd, and $\mathcal{T}$ be the Teichmuller set. Then

(a) the multiset $\mathcal{T}+\mathcal{T}+\mathcal{T}=\{X+Y+Z \mid X, Y, Z \in \mathcal{T}\}$ contains each element of $-\mathcal{T}$ with multiplicity one and the elements outside $-\mathcal{T}$ with multiplicity $2^{m}+1$;

(b) the multiset $\mathcal{T}+\mathcal{T}-\mathcal{T}=\{X+Y-Z \mid X, Y, Z \in \mathcal{T}\}$ contains each element of $\mathcal{T}$ with multiplicity $2^{m+1}-1$ and the elements outside $\mathcal{T}$ with multiplicity $2^{m}-1$.

Proof Here we only give the proof of (a), since a similar argument will prove (b). We need to investigate the number of solutions of the following equation:

$$
X+Y+Z=C,
$$


where $X, Y, Z \in \mathcal{T}$ and $C \in R$. The element $C$ can be uniquely expressed as $C=$ $A+2 B$, where $A, B \in \mathcal{T}$.

First, if $C \in-\mathcal{T}$, i.e., $A=B$, then we have $X+Y+Z+A=0$, implying $X=Y=$ $Z=A$. So the multiset $\mathcal{T}+\mathcal{T}+\mathcal{T}$ contains each element of $-\mathcal{T}$ with multiplicity one.

Next, if $C \notin-\mathcal{T}$, i.e., $A \neq B$, we split our consideration into two cases $A=0$ and $A \neq 0$.

When $A=0$, without loss of generality, we consider the equation

$$
X+Y+Z=2 \text {, }
$$

which is equivalent to the following system of binary equations:

$$
\left\{\begin{array}{l}
x+y+z=0 \\
x y+x z+y z=1
\end{array}\right.
$$

by Lemma B.1. From this system we have

$$
x^{2}+y^{2}+x y+1=0 .
$$

Assuming that $x=0$, we get one solution

$$
(x, y, z)=(0,1,1) .
$$

Assuming that $x \neq 0$ and $y=t x$ for some $t \in \mathbb{F}_{2^{m}}$, we obtain

$$
\left(t^{2}+t+1\right) x^{2}+1=0,
$$

implying

$$
(x, y, z)=\left(\frac{1}{\sqrt{t^{2}+t+1}}, \frac{t}{\sqrt{t^{2}+t+1}}, \frac{t+1}{\sqrt{t^{2}+t+1}}\right) .
$$

Hence, there are totally $2^{m}+1$ solutions for Eq. (6).

When $A \neq 0$, without loss of generality, we only need to investigate the equation

$$
X+Y+Z=1+2 B,
$$

where $B \in \mathcal{T}$ and $B \neq 1$. By Lemma B.1, Eq. (8) is equivalent to the following system:

$$
\left\{\begin{array}{l}
x+y+z=1 \\
x y+x z+y z=b^{2}
\end{array}\right.
$$

from which we have

$$
x^{2}+x y+y^{2}+x+y+b^{2}=0 .
$$

Now let $x=(1+b) u+b$ and $y=(1+b) v+b$. Then the above equation becomes

$$
u^{2}+u v+v^{2}+u+v=0
$$

so without loss of generality, we can assume that $b=0$ in Eq. (10). 
Assuming that $x=0$, we get two solutions $(x, y, z)=(0,0,1)$ and $(x, y, z)=$ $(0,1,0)$.

Assuming that $x \neq 0$ and $y=t x$, for some $t \in \mathbb{F}_{2^{m}}$, we have

$$
\left(t^{2}+t+1\right) x^{2}+(t+1) x=0,
$$

implying

$$
(x, y, z)=\left(\frac{t+1}{t^{2}+t+1}, \frac{t^{2}+t}{t^{2}+t+1}, \frac{t}{t^{2}+t+1}\right) .
$$

If $t=1$, there is only one solution $(x, y, z)=(0,0,1)$. Hence, there are totally $2^{m}+1$ solutions for Eq. (10).

The above lemma immediately implies the following result.

Corollary B.1 Let $R=G R(4, m), m$ odd, and $\mathcal{T}$ be the Teichmuller set.

(a) The multiset $\mathcal{T}+\mathcal{T}+\mathcal{T}+\mathcal{T}=\{X+Y+Z+W: X, Y, Z, W \in \mathcal{T}\}$ contains 0 with multiplicity $2^{m}$, the elements of $2 R \backslash\{0\}$ with multiplicity $2^{m}\left(2^{m}+1\right)$, and the elements outside $2 R$ with multiplicity $2^{2 m}$.

(b) The multiset $\mathcal{T}+\mathcal{T}-\mathcal{T}-\mathcal{T}=\{X+Y-Z-W: X, Y, Z, W \in \mathcal{T}\}$ contains 0 with multiplicity $\left(2^{m+1}-1\right) 2^{m}$, the elements of $2 R \backslash\{0\}$ with multiplicity $\left(2^{m}-1\right) 2^{m}$, and the elements outside $2 R$ with multiplicity $2^{2 m}$.

(c) The multiset $\mathcal{T}+\mathcal{T}+\mathcal{T}-\mathcal{T}=\{X+Y+Z-W: X, Y, Z, W \in \mathcal{T}\}$ contains the elements of $2 R$ with multiplicity $2^{2 m}$, the elements of $(\mathcal{T}+\mathcal{T}) \backslash 2 R$ with multiplicity $\left(2^{m}+1\right) 2^{m}$, and the elements outside $\mathcal{T}+\mathcal{T}$ with multiplicity $\left(2^{m}-1\right) 2^{m}$.

Recall the definition of the sets $\mathcal{E}_{0}, \ldots, \mathcal{E}_{9}$ in Sect. 3.

Corollary B.2 Let $q=2^{m}$ where $m \geq 3$ is odd, and $G=\mathbb{Z}_{4} \times R \times \mathbb{F}_{q}$. Then the sets $\mathcal{E}_{0}, \ldots, \mathcal{E}_{9}$ form a partition of $G$.

Proof Using (b) of Lemma B.2, we can check that the elements whose values at the first coordinate equal 0 appearing in $\mathcal{E}_{0}, \mathcal{E}_{1}, \mathcal{E}_{4}, \mathcal{E}_{5}, \mathcal{E}_{8}, \mathcal{E}_{9}$ indeed form a partition of $\{0\} \times R \times \mathbb{F}_{q}$. The case for the elements whose values at the first coordinate equal 2 can be verified using (c) of Lemma B.2. The cases for the elements whose values at the first coordinate equal 1 or 3 can be verified using (a) of Lemma B. 2 similarly.

Let $\operatorname{tr}(x)$ denote the trace function from $\mathbb{F}_{2^{m}}$ to $\mathbb{F}_{2}$. The following well-known simple result can be found in [18, p. 278].

Lemma B.4 Let $m$ be a positive integer. The quadratic equation $x^{2}+x+a=0$, $a \in \mathbb{F}_{2^{m}}$, has two roots in $\mathbb{F}_{2^{m}}$ if $\operatorname{tr}(a)=0$ and no roots in $\mathbb{F}_{2^{m}}$ if $\operatorname{tr}(a)=1$.

Let $f_{a}(x)=x^{3}+x+a$ and set

$$
M_{i}=\left\{a \in \mathbb{F}_{2^{m}}, a \neq 0 \mid f_{a}(x)=0 \text { has precisely } i \text { solutions in } \mathbb{F}_{2^{m}}\right\}
$$


for $i=0,1,3$. The exact values of the three numbers $\left|M_{0}\right|,\left|M_{1}\right|,\left|M_{3}\right|$ have been computed in the appendix of [16]:

$$
\begin{aligned}
& \left|M_{0}\right|=\frac{q+1}{3}, \\
& \left|M_{1}\right|=\frac{q}{2}-1, \quad \text { and } \\
& \left|M_{3}\right|=\frac{q-2}{6} .
\end{aligned}
$$

Lemma B.5 Let $m$ be a positive integer, and $f_{a}(x)=x^{3}+x+$ a for some $a \in \mathbb{F}_{2^{m}}$.

(a) If $a=0$, then $f_{a}(x)$ has two zeroes $x=0$ and 1 in $\mathbb{F}_{2^{m}}$.

(b) If $a=b+b^{-1}$ for some $b \in \mathbb{F}_{2^{m}} \backslash\{0,1\}$, then $f_{a}(x)$ has one and only one zero in $\mathbb{F}_{2^{m}}$.

(c) If $a=b^{-1}+b^{-3}$ for some $b \in \mathbb{F}_{2^{m}} \backslash\{0,1\}$ satisfying $\operatorname{tr}(b)=1$, then $f_{a}(x)$ has three distinct zeroes in $\mathbb{F}_{2^{m}}$.

(d) If a satisfies none of the above conditions, then $f_{a}(x)$ is irreducible over $\mathbb{F}_{2^{m}}$.

Proof (a) is immediate. One finds in the literature (see [5, p. 169]) that $f_{a}(x)=0$ has a unique solution in $\mathbb{F}_{2^{m}}$ if and only if $\operatorname{tr}(1 / a)=0$. If $a=b+b^{-1}$ for some $b \in \mathbb{F}_{2^{m}} \backslash\{0,1\}$, then

$$
\operatorname{tr}\left(\frac{1}{b+b^{-1}}\right)=\operatorname{tr}\left(\frac{b}{b^{2}+1}\right)=\operatorname{tr}\left(\frac{b}{b+1}+\left(\frac{b}{b+1}\right)^{2}\right)=0
$$

It is easy to check that the size of the set $\left\{b+b^{-1} \mid b \in \mathbb{F}_{2^{m}} \backslash\{0,1\}\right\}$ is equal to $\left|M_{1}\right|$. Then (b) follows.

If $a=b^{-1}+b^{-3}$ for some $b \in \mathbb{F}_{2^{m}} \backslash\{0,1\}$ satisfying $\operatorname{tr}(b)=1$, then we have

$$
\operatorname{tr}\left(\frac{1}{b^{-1}+b^{-3}}\right)=\operatorname{tr}\left(b+\frac{b}{b^{2}+1}\right)=\operatorname{tr}(b)=1 .
$$

Since $b^{-1}$ is already a zero of $f_{a}(x)$, we see that $f_{a}(x)$ must have three distinct zeroes in $\mathbb{F}_{2^{m}}$. The next thing is to check that the cardinality of the set $\left\{b^{-1}+b^{-3} \mid\right.$ $\left.b \in \mathbb{F}_{2^{m}} \backslash\{0,1\}, \operatorname{tr}(b)=1\right\}$ is the same as $\left|M_{3}\right|$. Suppose that $b^{-1}+b^{-3}=c^{-1}+c^{-3}$ for some $b \in \mathbb{F}_{2^{m}} \backslash\{0,1\}$ satisfying $\operatorname{tr}(b)=1$ and $c \in \mathbb{F}_{2^{m}} \backslash\{0,1\}$. We see that

$$
\begin{aligned}
b^{-1}+b^{-3} & =c^{-1}+c^{-3} \\
& \Longleftrightarrow \quad(b+c)\left(b^{2} c^{2}+b^{2}+b c+c^{2}\right)=0 \\
& \Longleftrightarrow \quad c=b \text { or } c^{2}+\left(b /\left(b^{2}+1\right)\right) c+b^{2} /\left(b^{2}+1\right)=0 .
\end{aligned}
$$

Because

$$
\operatorname{tr}\left(\frac{b^{2} /\left(b^{2}+1\right)}{b^{2} /\left(b^{2}+1\right)^{2}}\right)=\operatorname{tr}\left(b^{2}+1\right)=0,
$$


the equation $c^{2}+\left(b /\left(b^{2}+1\right)\right) c+b^{2} /\left(b^{2}+1\right)=0$ has two distinct zeroes in $\mathbb{F}_{2^{m}}$ by Lemma B.4. By the symmetry of the equation $b^{2} c^{2}+b^{2}+b c+c^{2}=0$, the value $\operatorname{tr}(c)$ must equal 1. Now it follows that the size of the set $\left\{b^{-1}+b^{-3} \mid b \in\right.$ $\left.\mathbb{F}_{2^{m}} \backslash\{0,1\}, \operatorname{tr}(b)=1\right\}$ is $(q-2) / 6=\left|M_{3}\right|$, finishing the prove of (c). Then (d) is immediate.

Throughout the rest of this section, we always assume that $q=2^{m}$ where $m \geq 3$ is odd.

Lemma B.6 Let $W \in \mathcal{T}$. The number of solutions to the system of equations

$$
\left\{\begin{array}{l}
X+Y-Z=W+2, \\
x^{3}+y^{3}+z^{3}=w^{3}+e
\end{array}\right.
$$

is (a) 1 when $e=0$, (b) 2 when $e \in M_{1}$, and (c) 0 when $e \in M_{0} \cup M_{3}$.

Proof Using the fact that $X+Y=(\sqrt{X}+\sqrt{Y})^{2}+2 \sqrt{X Y}$, the first equation $X+Y=$ $2+Z+W$ translates to the following two equations over $\mathbb{F}_{2^{m}}$ :

$$
x+y=z+w, \quad x y=z w+1 .
$$

Now we compute

$$
\begin{aligned}
e & =x^{3}+y^{3}+z^{3}+w^{3} \\
& =(x+y)^{3}+x y(x+y)+z^{3}+w^{3} \\
& =(z+w)^{3}+(z w+1)(z+w)+z^{3}+w^{3} \\
& =z+w .
\end{aligned}
$$

Hence, $z=e+w$ and $y=e+x$. Plugging them into $x y=z w+1$, we get $(x+z)^{2}+$ $e(x+z)+1=0$. When $e=0$, we get $x=1+z=e+w+1$, so this system only has one solution. When $e \neq 0$, we have $(x+z)^{2} / e^{2}+(x+z) / e+1 / e^{2}=0$. This equation has no or two solutions depending on whether $\operatorname{tr}(1 / e)=1$ or not.

Lemma B.7 Let $A, B \in \mathcal{T}$ and $B \neq 0$. The number of solutions to the system of equations

$$
\left\{\begin{array}{l}
X+Y-Z=A+2 B \\
x^{3}+y^{3}+z^{3}=a^{3}+b^{3} e
\end{array}\right.
$$

is (a) 1 when $e=0$, (b) 2 when $e \in M_{1}$, and (c) 0 when $e \in M_{0} \cup M_{3}$.

Similarly, we get the following result.

Lemma B.8 Let $A, B \in \mathcal{T}$ and $B \neq 0$. The number of solutions to the system

$$
\left\{\begin{array}{l}
X+Y+Z=-A+2 B, \\
x^{3}+y^{3}+z^{3}=a^{3}+b^{3} e
\end{array}\right.
$$

is (a) 3 when $e=0$, (b) 0 when $e \in M_{0} \cup M_{1}$, and (c) 6 when $e \in M_{3}$. 
The following corollaries can be easily verified from the proof of the above lemmas.

Corollary B.3 Let $0 \neq B \in \mathcal{T}$. The number of solutions to the system of equations

$$
\left\{\begin{array}{l}
X+Y-Z-W=2 B \\
x^{3}+y^{3}+z^{3}+w^{3}=b^{3} e
\end{array}\right.
$$

is (a) $2^{m}$ when $e=0$, (b) $2^{m+1}$ when $e \in M_{1}$, and (c) 0 when $e \in M_{0} \cup M_{3}$.

Corollary B.4 Let $0 \neq B \in \mathcal{T}$. The number of solutions to the system

$$
\left\{\begin{array}{l}
X+Y+Z+W=2 B \\
x^{3}+y^{3}+z^{3}+w^{3}=b^{3} e
\end{array}\right.
$$

is (a) $3 \cdot 2^{m}$ when $e=0$, (b) 0 , when $e \in M_{0} \cup M_{1}$, and (c) $6 \cdot 2^{m}$ when $e \in M_{3}$.

\section{Appendix C}

Throughout this section, we always assume that $q=2^{m}$ where $m \geq 3$ is odd. For $(a, b) \in G \times \mathbb{F}_{q}$, the $\operatorname{sum} \xi(a, b)$ has the following properties:

(a) If $b \neq 0$, then $\xi(a, b)=\xi\left(a B^{-1 / 3}, 1\right)$;

(b) If $U, V \in \mathcal{T}, W \in \mathcal{T}^{*}$, then $\xi(U+2 V, b)=\xi\left(U W+2 V W, b w^{3}\right)$.

Now we fix some notation. Let $a$ and $c$ be elements of $R$ and write them as $a=$ $U+2 V$ and $c=S+2 T$ where $U, V, S, T \in \mathcal{T}$. For convenience, we define $\eta_{a}$ as $\eta_{a}=\xi(a, 1)$. Let $u$ be the projection of $a$ modulo 2 in $\mathbb{F}_{q}$. Set

$$
f_{u}(z)=z^{2}+u^{2} z+\sqrt{z}+u
$$

and let $F_{u}$ be the zeros of $f_{u}(z)$ in $\mathbb{F}_{q}$. Also set

$$
h_{u}(z)=f_{u}(z)-u=z^{2}+u^{2} z+\sqrt{z}
$$

and let $H_{u}$ be the zeros of $h_{u}(z)$ in $\mathbb{F}_{q}$. It is easy to see $u^{2} \in F_{u}$ and

$$
F_{u}=\left\{x+u^{2} \mid x \in H_{u}\right\}
$$

so $\left|F_{u}\right|=\left|H_{u}\right|$. For each $x \in H_{u}$, we have $\operatorname{tr}(u x)=\operatorname{tr}\left(u^{2} x^{2}\right)=\operatorname{tr}\left(x^{3}+x^{3 / 2}\right)=0$, which yields that $\operatorname{tr}(u y)=\operatorname{tr}\left(u^{3}\right)$ for each $y \in F_{u}$. For $X, Y \in \mathcal{T}$, we see that

$$
X+Y=(\sqrt{X}+\sqrt{Y})^{2}+2 \sqrt{X Y} .
$$

The element $(\sqrt{X}+\sqrt{Y})^{2} \in \mathcal{T}$ will be denoted as $X \oplus Y$ in the following. Here come some useful results involving $\eta_{a}$. 
Lemma C.1 Let $a \in R$, and let $u$ be the projection of a modulo 2 in $\mathbb{F}_{q}$. The exponential sum $\eta_{a}$ satisfies:

$$
\begin{array}{ll}
\eta_{a}^{2}=2^{m} \sum_{\substack{Z \in \mathcal{T} \\
f_{u}(z)=0}} i^{\mathrm{T}\left(a Z+2 Z^{3}\right)}, \quad \eta_{a} \overline{\eta_{a}}=2^{m} \sum_{\substack{Z \in \mathcal{T} \\
h_{u}(z)=0}} i^{\mathrm{T}\left(a Z+2 Z^{3}\right)}, \\
\eta_{a}^{4}=2^{m}(-1)^{\operatorname{tr}\left(u^{3}\right)}\left|F_{u}\right| \eta_{a} \overline{\eta_{a}}, \quad\left(\eta_{a} \overline{\eta_{a}}\right)^{2}=2^{m}\left|F_{u}\right| \eta_{a} \overline{\eta_{a}}, \quad \eta_{a}^{3} \overline{\eta_{a}}=2^{m}\left|F_{u}\right| \eta_{a}^{2} .
\end{array}
$$

Proof We first compute

$$
\begin{aligned}
\eta_{a}^{2} & =\sum_{X \in \mathcal{T}} \sum_{Y \in \mathcal{T}} i^{\mathrm{T}\left(a(X+Y)+2\left(X^{3}+Y^{3}\right)\right)}=\sum_{Y \in \mathcal{T}} \sum_{Z \in \mathcal{T}} i^{\mathrm{T}\left(a(Y \oplus Z+Y)+2\left((Y \oplus Z)^{3}+Y^{3}\right)\right)} \\
& =\sum_{Y \in \mathcal{T}} \sum_{Z \in \mathcal{T}} i^{T\left(a Z+2 Z^{3}+2\left(a Y+a \sqrt{Y Z}+Y^{2} Z+Z^{2} Y+Z^{3}\right)\right)} \\
& =\sum_{Z \in \mathcal{T}} i^{\mathrm{T}\left(a Z+2 Z^{3}\right)} \sum_{y \in \mathbb{F}_{q}}(-1)^{\operatorname{tr}\left(y\left(z^{2}+u^{2} z+\sqrt{z}+u\right)\right)} \\
& =2^{m} \sum_{\substack{Z \in \mathcal{T} \\
f_{u}(z)=0}} i^{\mathrm{T}\left(a Z+2 Z^{3}\right)} .
\end{aligned}
$$

A similar analysis gives

$$
\eta_{a} \overline{\eta_{a}}=2^{m} \sum_{\substack{Z \in \mathcal{T} \\ h_{u}(z)=0}} i^{\mathrm{T}\left(a Z+2 Z^{3}\right)} .
$$

Then

$$
\begin{aligned}
\eta_{a}^{4} & =2^{2 m} \sum_{\substack{Z \in \mathcal{T} \\
f_{u}(z)=0}} \sum_{\substack{W \in \mathcal{T} \\
f_{u}(w)=0}} i^{\mathrm{T}\left(a(Z+W)+2\left(Z^{3}+W^{3}\right)\right)} \\
& =2^{2 m} \sum_{\substack{Z \in \mathcal{T} \\
f_{u}(z)=0}} \sum_{\substack{W \in \mathcal{T} \\
h_{u}(w)=0}} i^{\mathrm{T}\left(a(Z+Z \oplus W)+2\left(Z^{3}+(Z \oplus W)^{3}\right)\right)} \\
& =2^{2 m} \sum_{\substack{W \in \mathcal{T} \\
h_{u}(w)=0}} i^{\mathrm{T}\left(a W+2 W^{3}\right)} \sum_{\substack{Z \in \mathcal{T} \\
f_{u}(z)=0}}(-1)^{\operatorname{tr}\left(u z+w\left(z^{2}+u^{2} z+\sqrt{z}\right)\right)} \\
& =2^{2 m} \sum_{\substack{W \in \mathcal{T} \\
h_{u}(w)=0}} i^{\mathrm{T}\left(a W+2 W^{3}\right)} \sum_{\substack{Z \in \mathcal{T} \\
f_{u}(z)=0}}(-1)^{\operatorname{tr}(u(z+w))} \\
& =2^{m}(-1)^{\operatorname{tr}\left(u^{3}\right)}\left|F_{u}\right| \eta_{a} \overline{\eta_{a}} .
\end{aligned}
$$

The same calculations as above will prove the remaining equations. 
Lemma C.2 Let $c \in R \backslash 2 R$ and $d \in \mathbb{F}_{q}$. Then c can be expressed uniquely as $c=$ $F-G$ where $F, G \in \mathcal{T}$. We have

$$
\mathbf{E}(c, d)= \begin{cases}2^{3 m+4}\left(3 \cdot 2^{m-1}-1\right) & \text { if } d=f^{3}+g^{3}, \\ 2^{3 m+4}\left(2^{m-1}-1\right) & \text { if } d \neq f^{3}+g^{3},\end{cases}
$$

where $f, g$ are the projections of $F, G$ modulo 2 in $\mathbb{F}_{q}$.

Proof It is direct to check that

$$
s t^{2}+s^{-1} t^{4}+s^{3}=f^{3}+g^{3}
$$

and

$$
\operatorname{tr}\left(s^{-3}\left(f^{3}+g^{3}\right)\right)=1 .
$$

Let $X \in \mathcal{T}$ and $B \in \mathcal{T}^{*}$. We first compute

$$
\begin{aligned}
\mathcal{U} & :=i^{\mathrm{T}(X c)} \sum_{Y \in \mathcal{T}} \eta_{(X+2 Y) B^{-1 / 3}} \overline{\eta_{(X+2 Y) B^{-1 / 3}}}(-1)^{\operatorname{tr}(y s)} \\
& =2^{m} i^{\mathrm{T}(X c)} \sum_{Y \in \mathcal{T}} \sum_{\substack{Z \in \mathcal{T} \\
h_{x^{\prime}}(z)=0}} i^{\mathrm{T}\left((X+2 Y) B^{-1 / 3} Z+2 Z^{3}\right)}(-1)^{\operatorname{tr}(y s)} \\
& =2^{m} i^{\mathrm{T}(X c)} \sum_{\substack{Z \in \mathcal{T} \\
h_{x^{\prime}}(z)=0}} i^{\mathrm{T}\left(X B^{-1 / 3} Z+2 Z^{3}\right)} \sum_{Y \in \mathcal{T}}(-1)^{\operatorname{tr}\left(y\left(b^{-1 / 3} z+s\right)\right)} \\
& = \begin{cases}2^{2 m}(-1)^{\operatorname{tr}\left(b\left(f^{3}+g^{3}\right)\right)} & \text { if } X=B^{\frac{1}{2}} S^{\frac{1}{2}} \oplus B^{\frac{1}{4}} S^{-\frac{1}{4}}, \\
0 & \text { otherwise, }\end{cases}
\end{aligned}
$$

where $x^{\prime}=x b^{-1 / 3}$. Notice that $B^{\frac{1}{2}} S^{\frac{1}{2}} \oplus B^{\frac{1}{4}} S^{-\frac{1}{4}}=0$ if $b=s^{-3}$.

Let $x, s \in \mathbb{F}_{q}^{*}$ and $b \in \mathbb{F}_{q}^{*} \backslash\left\{s^{-3}\right\}$. We consider the set $H_{x}$. Immediately, 0 is a root of $h_{x}(z)$. The equation $h_{x}(z)=0$ is equivalent to

$$
\left(h_{x}(z)\right)^{2}=z\left(z^{3}+x^{4} z+1\right)=0 .
$$

Replace $z$ with $x^{2} w$; then $z^{3}+x^{4} z+1=0$ becomes $w^{3}+w+x^{-6}=0$.

If

$$
x=(b s)^{1 / 2}+\left(b s^{-1}\right)^{1 / 4},
$$

then

$$
x^{6}=b^{1 / 2} s^{3 / 2}+b s^{3}+b^{-1 / 2} s^{-3 / 2}+1
$$

and

$$
x^{-6}=\left(1+b^{-1 / 2} s^{-3 / 2}\right)^{-1}+\left(1+b^{-1 / 2} s^{-3 / 2}\right)^{-3} \text {. }
$$


Using Lemma B.5, we see that if

$$
\operatorname{tr}\left(1+b^{-1 / 2} s^{-3 / 2}\right)=0
$$

then

$$
\operatorname{tr}\left(x^{6}\right)=\operatorname{tr}\left(1+b^{-1} s^{-3}\right)=0,
$$

implying

$$
\left|F_{b^{\frac{1}{6}} s^{\frac{1}{2}}+b^{-\frac{1}{12}} s^{-\frac{1}{4}}}\right|=\left|H_{b^{\frac{1}{6}} s^{\frac{1}{2}}+b^{-\frac{1}{12}} s^{-\frac{1}{4}}}\right|=2 ;
$$

otherwise, we have

$$
\left|F_{b^{\frac{1}{6}} s^{\frac{1}{2}}+b^{-\frac{1}{12}} s^{-\frac{1}{4}}}\right|=\left|H_{b^{\frac{1}{6}} s^{\frac{1}{2}}+b^{-\frac{1}{12}} s^{-\frac{1}{4}}}\right|=4 .
$$

So the expression

$$
\left(2(-1)^{\operatorname{tr}\left(1+b^{-1} s^{-3}\right)}+6\right) \mid F_{b^{\frac{1}{6}} s^{\frac{1}{2}}+b^{-\frac{1}{12}} s^{-\frac{1}{4}} \mid}
$$

is always equal to 16 .

The sum $\mathbf{E}(c, d)$ will be divided into four parts and computed separately with the assistance of Lemma C.1. We first compute

$$
\begin{aligned}
& \mathbf{E}(c, d)_{1} \\
& =\sum_{a \in R^{*}} \sum_{b \in \mathbb{F}_{q}^{*}}\left(\xi^{4}(a, b)+\overline{\xi^{4}(a, b)}+6 \xi^{2}(a, b) \overline{\xi^{2}(a, b)}\right) i^{\mathrm{T}(a c+2 b d)} \\
& =\sum_{X \in \mathcal{T}^{*}} \sum_{Y \in \mathcal{T}} \sum_{b \in \mathbb{F}_{q}^{*}}\left(\xi^{4}(X+2 Y, b)+\overline{\xi^{4}(X+2 Y, b)}\right. \\
& +6 \xi^{2}(X+2 Y, b) \overline{\left.\xi^{2}(X+2 Y, b)\right)} i^{\mathrm{T}((X+2 Y) c+2 b d)} \\
& =\sum_{X \in \mathcal{T}^{*}} \sum_{Y \in \mathcal{T}} \sum_{b \in \mathbb{F}_{q}^{*}}\left(\eta_{(X+2 Y) B^{-\frac{1}{3}}}^{4}+\overline{\eta_{(X+2 Y) B^{-\frac{1}{3}}}^{4}}\right. \\
& +6 \eta_{(X+2 Y) B^{-\frac{1}{3}}} \overline{\eta_{(X+2 Y) B^{-\frac{1}{3}}}^{2}} i^{\mathrm{T}((X+2 Y) c+2 b d)} \\
& =2^{m} \sum_{X \in \mathcal{T}^{*}} \sum_{b \in \mathbb{F}_{q}^{*}}(-1)^{\operatorname{tr}(b d)}\left(2(-1)^{\operatorname{tr}\left(x^{3} b^{-1}\right)}+6\right)\left|F_{x b^{-\frac{1}{3}}}\right| \mathcal{U} \\
& =2^{3 m} \sum_{b \in \mathbb{F}_{q}^{*} \backslash\left\{s^{-3}\right\}}\left[\left(2(-1)^{\operatorname{tr}\left(1+b^{-1} s^{-3}\right)}+6\right)\left|F_{b^{\frac{1}{6}} s^{\frac{1}{2}}+b^{-\frac{1}{12}} s^{-\frac{1}{4}}}\right|\right](-1)^{\operatorname{tr}\left(b\left(f^{3}+g^{3}+d\right)\right)} \\
& =2^{3 m+4} \sum_{b \in \mathbb{F}_{q}^{*} \backslash\left\{s^{-3}\right\}}(-1)^{\operatorname{tr}\left(b\left(f^{3}+g^{3}+d\right)\right)} .
\end{aligned}
$$


Next,

$$
\begin{aligned}
\mathbf{E}(c, d)_{2}= & \sum_{X \in \mathcal{T}^{*}} \sum_{Y \in \mathcal{T}}\left(\xi^{4}(X+2 Y, 0)+\overline{\xi^{4}(X+2 Y, 0)}\right. \\
& \left.+6 \xi^{2}(X+2 Y, 0) \overline{\xi^{2}(X+2 Y, 0)}\right) i^{\mathrm{T}((X+2 Y) c)} \\
= & \sum_{X \in \mathcal{T}^{*}} \sum_{Y \in \mathcal{T}^{2 m}}\left(2^{2 m} i^{\mathrm{T}(2)}+2^{2 m} i^{-\mathrm{T}(2)}+6 \cdot 2^{2 m}\right) i^{\mathrm{T}((X+2 Y) c)} \\
= & 2^{2 m+2} \sum_{X \in \mathcal{T}^{*}} i^{\mathrm{T}(X c)} \sum_{Y \in \mathcal{T}}(-1)^{\operatorname{tr}(y s)} \\
= & 0 .
\end{aligned}
$$

Then,

$$
\begin{aligned}
\mathbf{E}(c, d)_{3} & =\sum_{Y \in \mathcal{T}} \sum_{b \in \mathbb{F}_{q}^{*}}\left(\xi^{4}(2 Y, b)+\overline{\xi^{4}(2 Y, b)}+6 \xi^{2}(2 Y, b) \overline{\xi^{2}(2 Y, b)}\right) i^{\mathrm{T}(2 Y c+2 b d)} \\
& =\sum_{Y \in \mathcal{T}} \sum_{b \in \mathbb{F}_{q}^{*}}\left(2^{2 m+4}\left(1+(-1)^{\operatorname{tr}\left(y b^{-1 / 3}+1\right)}\right)\right)(-1)^{\operatorname{tr}(y s+b d)} \\
& =2^{2 m+4} \sum_{b \in \mathbb{F}_{q}^{*}}(-1)^{\operatorname{tr}(b d+1)} \sum_{Y \in \mathcal{T}}(-1)^{\operatorname{tr}\left(y\left(b^{-1 / 3}+s\right)\right)} \\
& =2^{3 m+4}(-1)^{\operatorname{tr}\left(s^{-3} d+1\right)} .
\end{aligned}
$$

At last,

$$
\mathbf{E}(c, d)_{4}=\xi^{4}(0,0)+\overline{\xi^{4}(0,0)}+6 \xi^{2}(0,0) \overline{\xi^{2}(0,0)}=2^{4 m+3} .
$$

Adding $\mathbf{E}(c, d)_{1}, \mathbf{E}(c, d)_{2}, \mathbf{E}(c, d)_{3}$, and $\mathbf{E}(c, d)_{4}$ up will complete the proof.

Lemma C.3 Let $c \in R \backslash 2 R$ and $d \in \mathbb{F}_{q}$. There exist $F, G \in \mathcal{T}$ such that $c=F+G$ with $F \neq G$. We have

$$
\mathbf{F}(c, d)= \begin{cases}2^{3 m+2}\left(3 \cdot 2^{m-1}-1\right) & \text { if } d=f^{3}+g^{3}, \\ 2^{3 m+2}\left(2^{m-1}-1\right) & \text { if } d \neq f^{3}+g^{3}\end{cases}
$$

where $f, g$ are the projections of $F, G$ modulo 2 in $\mathbb{F}_{q}$.

Proof It is easy to check that

$$
s t^{2}+s^{3}=f^{3}+g^{3} .
$$

Let $X \in \mathcal{T}$ and $B \in \mathcal{T}^{*}$. We see

$$
\begin{aligned}
\mathcal{V}:= & i^{\mathrm{T}(X)} \sum_{Y \in \mathcal{T}}\left(\eta_{(X+2 Y) B^{-1 / 3}}^{3} \overline{\eta_{(X+2 Y) B^{-1 / 3}}}\right. \\
& \left.+\eta_{(X+2 Y) B^{-1 / 3}} \overline{\left.\eta_{(X+2 Y) B^{-1 / 3}}^{3}\right)}\right)(-1)^{\operatorname{tr}(y)}
\end{aligned}
$$




$$
\begin{aligned}
& =2^{2 m}\left|K_{x b^{-1 / 3}}\right| i^{\mathrm{T}(X)} \sum_{\substack{Z \in \mathcal{T} \\
f_{X^{\prime}}(z)=0}}\left(i^{\mathrm{T}\left(X B^{-1 / 3} Z+2 Z^{3}\right)}\right. \\
& \left.+i^{-\mathrm{T}\left(X B^{-1 / 3} Z+2 Z^{3}\right)}\right) \sum_{Y \in \mathcal{T}}(-1)^{\operatorname{tr}\left(v\left(b^{-1 / 3} z+1\right)\right)} \\
& = \begin{cases}2^{3 m}\left|K_{b^{1 / 6}}\right|\left(1+(-1)^{\operatorname{tr}(b)}\right) & \text { if } U=B^{1 / 2}, \\
2^{3 m} \mid K_{b^{1 / 6}+b^{-1 / 3} \mid\left(-1+(-1)^{\operatorname{tr}(b)}\right)} & \text { if } U=1 \oplus B^{1 / 2}, \\
0 & \text { otherwise, }\end{cases}
\end{aligned}
$$

where $x^{\prime}=x b^{-1 / 3}$.

We first calculate

$$
\begin{aligned}
& \mathbf{F}(c, d)_{1}=\sum_{X \in \mathcal{T}^{*}} \sum_{Y \in \mathcal{T}} \sum_{b \in \mathbb{F}_{q}^{*}}\left(\xi^{3}(X+2 Y, b) \overline{\xi(X+2 Y, b)}\right. \\
& \left.+\xi(X+2 Y, b) \overline{\xi^{3}(X+2 Y, b)}\right) i^{\mathrm{T}((X+2 Y) c+2 b d)} \\
& =\sum_{X \in \mathcal{T}^{*}} \sum_{Y \in \mathcal{T}} \sum_{b \in \mathbb{F}_{q}^{*}}\left(\xi^{3}\left((X+2 Y) S, b s^{3}\right) \overline{\xi\left((X+2 Y) S, b s^{3}\right)}\right. \\
& \left.+\xi\left((X+2 Y) S, b s^{3}\right) \overline{\xi^{3}\left((X+2 Y) S, b s^{3}\right)}\right) i^{\mathrm{T}\left((X+2 Y) S\left(1+2 S^{-1} T\right)+2 b d\right)} \\
& =\sum_{X \in \mathcal{T}^{*}} \sum_{Y \in \mathcal{T}} \sum_{b \in \mathbb{F}_{q}^{*}}\left(\xi^{3}(X+2 Y, b) \overline{\xi(X+2 Y, b)}\right. \\
& \left.+\xi(X+2 Y, b) \overline{\xi^{3}(X+2 Y, b)}\right) i^{\mathrm{T}\left((X+2 Y)\left(1+2 S^{-1} T\right)+2 b s^{-3} d\right)} \\
& =\sum_{X \in \mathcal{T}^{*}} \sum_{b \in \mathbb{F}_{q}^{*}} i^{\mathrm{T}\left(2 X S^{-1} T+2 b s^{-3} d\right)} \mathcal{V} \\
& =2^{3 m} \sum_{b \in \mathbb{F}_{q}^{*}}\left|K_{b^{1 / 6}}\right|(-1)^{\operatorname{tr}\left(b^{1 / 2} s^{-1} t+b s^{-3} d\right)}\left(1+(-1)^{\operatorname{tr}(b)}\right) \\
& +2^{3 m} \sum_{b \in \mathbb{F}_{q} \backslash\{0,1\}}\left|K_{b^{1 / 6}+b^{-1 / 3}}\right|(-1)^{\operatorname{tr}\left(b^{1 / 2} s^{-1} t+s^{-1} t+b s^{-3} d\right)}\left(-1+(-1)^{\operatorname{tr}(b)}\right) \\
& =2^{3 m+2}\left(\sum_{\substack{b \in \mathbb{F}^{*} \\
\operatorname{tr}(b)=0}}(-1)^{\operatorname{tr}\left(b\left(s^{-2} t^{2}+s^{-3} d\right)\right)}+\sum_{\substack{b \in \mathbb{F}_{q} \backslash\{0,1\} \\
\operatorname{tr}(b)=1}}(-1)^{\operatorname{tr}\left(s^{-1} t+b\left(s^{-2} t^{2}+s^{-3} d\right)\right)}\right) \\
& =2^{3 m+2}\left(1+(-1)^{\operatorname{tr}\left(s^{-3} d+1\right)}\right) \sum_{\substack{b \in \mathbb{F}_{q}^{*} \\
\operatorname{tr}(b)=0}}(-1)^{\operatorname{tr}\left(b\left(s^{-2} t^{2}+s^{-3} d\right)\right)} \\
& = \begin{cases}2^{3 m+3}\left(2^{m-1}-1\right) & \text { if } d=f^{3}+g^{3}, \\
-2^{3 m+2}\left(1+(-1)^{\operatorname{tr}\left(s^{-3} d+1\right)}\right) & \text { otherwise. }\end{cases}
\end{aligned}
$$


Next,

$$
\begin{aligned}
\mathbf{F}(c, d)_{2}= & \sum_{X \in \mathcal{T}^{*}} \sum_{Y \in \mathcal{T}}\left(\xi^{3}(X+2 Y, 0) \overline{\xi(X+2 Y, 0)}\right. \\
& \left.+\xi(X+2 Y, 0) \overline{\xi^{3}(X+2 Y, 0)}\right) i^{\mathrm{T}((X+2 Y) c)} \\
= & 2^{2 m} \sum_{X \in \mathcal{T}^{*}} \sum_{Y \in \mathcal{T}}\left(i^{\mathrm{T}\left(1+2 X^{-1} Y+(X+2 Y) c\right)}+i^{\mathrm{T}\left(-1-2 X^{-1} Y+(X+2 Y) c\right)}\right) \\
= & 2^{2 m}\left(\sum_{X \in \mathcal{T}^{*}} i^{\mathrm{T}(1+X c)}+\sum_{X \in \mathcal{T}^{*}} i^{\mathrm{T}(-1+X c)}\right) \sum_{Y \in \mathcal{T}}(-1)^{\operatorname{tr}\left(y\left(x^{-1}+s\right)\right)} \\
= & 2^{3 m}\left(i^{\mathrm{T}\left(2+2 s^{-1} t\right)}+i^{\mathrm{T}\left(2 s^{-1} t\right)}\right) \\
= & 0 .
\end{aligned}
$$

Then we calculate

$$
\begin{aligned}
\mathbf{F}(c, d)_{3} & =\sum_{Y \in \mathcal{T}} \sum_{b \in \mathbb{F}_{q}^{*}}\left(\xi^{3}(2 Y, b) \overline{\xi(2 Y, b)}+\xi(2 Y, b) \overline{\xi^{3}(2 Y, b)}\right) i^{\mathrm{T}(2 Y c+2 b d)} \\
& =2^{2 m+2} \sum_{Y \in \mathcal{T}} \sum_{b \in \mathbb{F}_{q}^{*}}\left(1+(-1)^{\operatorname{tr}\left(y b^{-1 / 3}+1\right)}\right)(-1)^{\operatorname{tr}(y s+b d)} \\
& =2^{2 m+2} \sum_{b \in \mathbb{F}_{q}^{*}}(-1)^{\operatorname{tr}(b d+1)} \sum_{Y \in \mathcal{T}}(-1)^{\operatorname{tr}\left(y\left(b^{-1 / 3}+s\right)\right)} \\
& =2^{3 m+2}(-1)^{\operatorname{tr}\left(s^{-3} d+1\right)}
\end{aligned}
$$

At last,

$$
\mathbf{F}(c, d)_{4}=\xi^{3}(0,0) \overline{\xi(0,0)}+\xi(0,0) \overline{\xi^{3}(0,0)}=2^{4 m+1}
$$

Adding $\mathbf{F}(c, d)_{1}, \mathbf{F}(c, d)_{2}, \mathbf{F}(c, d)_{3}$, and $\mathbf{F}(c, d)_{4}$ up will complete the proof.

The following result can be proved similarly as above.

Lemma C.4 Let $c \in 2 R$ and $d \in \mathbb{F}_{q}$. We have

$$
\mathbf{F}(c, d)= \begin{cases}2^{3 m+2}\left(3 \cdot 2^{m-1}-1\right) & \text { if } d=0 \\ 2^{3 m+2}\left(2^{m-1}-1\right) & \text { if } d \neq 0 .\end{cases}
$$

\section{References}

1. Abdukhalikov, K., Bannai, E., Suda, S.: Association schemes related to universally optimal configurations, Kerdock codes and extremal Euclidean line-sets. J. Comb. Theory, Ser. A 116(2), 434-448 (2009) 
2. Ballinger, B., Blekherman, G., Cohn, H., Giansiracusa, N., Kelly, E., Schürmann, A.: Experimental study of energy-minimizing point configurations on spheres. Exp. Math. 18(3), 257-283 (2009)

3. Bannai, E.: Subschemes of some association schemes. J. Algebra 144(1), 167-188 (1991)

4. Bannai, E., Ito, T.: Algebraic Combinatorics I: Association Schemes. Benjamin-Cummings, Menlo Park (1984)

5. Berlekamp, E.R.: Algebraic Coding Theory. McGraw-Hill, New York (1968)

6. Bonnecaze, A., Duursma, I.M.: Translates of linear codes over $Z_{4}$. IEEE Trans. Inf. Theory 43(4), 1218-1230 (1997)

7. Brouwer, A.E., Cohen, A.M., Neumaier, A.: Distance-Regular Graphs. Ergebnisse der Mathematik und Ihrer Grenzgebiete (3), vol. 18. Springer, Berlin (1989). Results in Mathematics, and Related Areas (3)

8. Davis, J.A., Xiang, Q.: Negative Latin square type partial difference sets in nonelementary Abelian 2-groups. J. Lond. Math. Soc. (2) 70(1), 125-141 (2004)

9. Hagita, M., Schmidt, B.: Bijections between group rings preserving character sums. Des. Codes Cryptogr. 24(3), 243-254 (2001)

10. Hammons, A.R. Jr., Kumar, P.V., Calderbank, A.R., Sloane, N.J.A., Solé, P.: The $\mathbf{Z}_{4}$-linearity of Kerdock, Preparata, Goethals, and related codes. IEEE Trans. Inf. Theory 40(2), 301-319 (1994)

11. Harada, M.: New 5-designs constructed from the lifted Golay code over $Z_{4}$. J. Comb. Des. 6(3), 225-229 (1998)

12. Helleseth, T., Kumar, P.V.: The algebraic decoding of the $Z_{4}$-linear Goethals codes. IEEE Trans. Inf. Theory 41(6, part 2), 2040-2048 (1995)

13. Helleseth, T., Zinoviev, V.: Codes with the same coset weight distributions as the $Z_{4}$-linear Goethals codes. IEEE Trans. Inf. Theory 47(4), 1589-1595 (2001)

14. Helleseth, T., Zinoviev, V.: On coset weight distributions of the $Z_{4}$-linear Goethals codes. IEEE Trans. Inf. Theory 47(5), 1758-1772 (2001)

15. Helleseth, T., Kumar, P.V., Shanbhag, A.: Codes with the same weight distributions as the Goethals codes and the Delsarte-Goethals codes. Des. Codes Cryptogr. 9(3), 257-266 (1996)

16. Kumar, P.V., Helleseth, T., Calderbank, A.R., Hammons, A.R. Jr.: Large families of quaternary sequences with low correlation. IEEE Trans. Inf. Theory 42(2), 579-592 (1996)

17. Liebler, R.A., Mena, R.A.: Certain distance-regular digraphs and related rings of characteristic 4. J. Comb. Theory, Ser. A 47(1), 111-123 (1988)

18. MacWilliams, F.J., Sloane, N.J.A.: The Theory of Error-Correcting Codes. North-Holland Mathematical Library, vol. 16. North-Holland, Amsterdam (1977)

19. Muzychuk, M.E.: V-rings of permutation groups with invariant metric. Ph.D. thesis, Kiev State University (1987)

20. Pasini, A., Yoshiara, S.: New distance regular graphs arising from dimensional dual hyperovals. Eur. J. Comb. 22(4), 547-560 (2001)

21. Shin, D.-J., Kumar, P.V., Helleseth, T.: An Assmus-Mattson-type approach for identifying 3-designs from linear codes over $Z_{4}$. Des. Codes Cryptogr. 31(1), 75-92 (2004)

22. Solé, P.: Four applications of $\mathbb{Z}_{4}$-codes. In: Fifth Conference on Discrete Mathematics and Computer Science (Spanish). Ciencias (Valladolid), vol. 23, pp. 37-40. Univ. Valladolid, Valladolid (2006). Secr. Publ. Intercamb. Ed. 